\title{
OPTIMAL CONTROL AND NUMERICAL ADAPTIVITY FOR ADVECTION-DIFFUSION EQUATIONS
}

\author{
Luca Dede ${ }^{1}$ And Alfio Quarteroni ${ }^{1,2}$
}

\begin{abstract}
We propose a general approach for the numerical approximation of optimal control problems governed by a linear advection-diffusion equation, based on a stabilization method applied to the Lagrangian functional, rather than stabilizing the state and adjoint equations separately. This approach yields a coherently stabilized control problem. Besides, it allows a straightforward a posteriori error estimate in which estimates of higher order terms are needless. Our a posteriori estimates stems from splitting the error on the cost functional into the sum of an iteration error plus a discretization error. Once the former is reduced below a given threshold (and therefore the computed solution is "near" the optimal solution), the adaptive strategy is operated on the discretization error. To prove the effectiveness of the proposed methods, we report some numerical tests, referring to problems in which the control term is the source term of the advection-diffusion equation.
\end{abstract}

Mathematics Subject Classification. 35J25, 49J20, 65N30, 76R50.

Received: February 9, 2005. Revised: April 22, 2005.

\section{INTRODUCTION}

Many physical problems can be modelled by linear advection-diffusion partial differential equations; this is the case for example if we want to forecast the distribution of a substance in a continuous medium, such as a pollutant in air or water. In this contest it is interesting to operate the source terms (e.g. the emission rate of pollutants) in order that the PDE solution approaches as closely as possible a desired distribution (or, otherwise said, the concentration of pollutant stands below a pre-assigned threshold). This aspect can be conveniently accommodated in the framework of the optimal control theory, for which we assume as control function the source term, while the "observation" is a function depending on the PDE solution. The classical approach to this kind of problems is based on the theory developed by Lions [10] (see also $[2,13,15,17]$ ), or, as complementary to the previous one, on the Lagrangian functional formalism [4]. By adopting the latter methodology, which is useful for practical problems, but it does not ensure the existence and uniqueness of solution (see [1]), we address a generic optimal control problem applied to an advection-diffusion equation. For its approximation we use an iterative method applied to the Galerkin-FE discretization of both state and adjoint equations. To get rid of numerical instabilities arising in the transport dominated regimes, we propose a stabilization on the Lagrangian

\footnotetext{
Keywords and phrases. Optimal control problems, partial differential equations, finite element approximation, stabilized Lagrangian, numerical adaptivity, advection-diffusion equations.

1 MOX-Dipartimento di Matematica "F. Brioschi", Politecnico di Milano, 20133, Milano, Italy. luca.dede@mate.polimi.it

2 École Polytechnique Fédérale de Lausanne (EPFL), FSB, Chaire de Modelisation et Calcul Scientifique (CMCS), Station 8, 1015, Lausanne, Switzerland. alfio.quarteroni@epfl.ch
} 
functional term, introducing the stabilized Lagrangian concept, rather than stabilizing separately the state and adjoint equations [16]. By this approach we achieve intrinsic coherence between the stabilized state and adjoint equations. Moreover, we propose a posteriori estimate of the error on the control problem, that we interpret as the error on the cost functional $[3,4]$, i.e. on the observation of the system. With this approach we treat the error contribution arising from the numerical discretization separately from the one arising from the iteration process, in view of operating the grid adaptation. More precisely, our a posteriori estimates stem from splitting the error on the cost functional into the sum of an iteration error plus a discretization error. Once the former is reduced below a given threshold (and therefore the computed solution is "near" the optimal solution), the adaptive strategy is operated on the discretization error.

We prove the effectiveness of the proposed method on a couple of test cases in which the advection-diffusion equation is associated to a pollution problem (atmospheric and hydraulic) for which the control term is the source term of the equation. In this contest the problems can be interpreted as emission control problems, e.g. the control of the rate of emission to keep the pollutant level in water (or in air) below a desired threshold.

An outline of this paper is as follows. In Section 1 we formulate an abstract control problem, for a linear advection-diffusion equation. In Section 2 we introduce the stabilized Lagrangian concept and provide a complete analysis of the stabilized control problem. In Section 3 we propose the separation of the iteration and discretization error, for which we provide the a posteriori error estimate; we also report a method to introduce the grid adaptivity in the contest of the iterative optimization and we propose a quadratic reconstruction method in order to compute the error indicator. In Section 4 we report some numerical results applied to two test problems, for which we show the effectiveness of the proposed methods.

\section{Optimal Control problem For adVECtion-Diffusion EQUATIONS}

We start by providing a general approach for the analysis of optimal control problems, that we will apply to the particular case of problems governed by a linear advection-diffusion equation.

\subsection{An abstract formulation for optimal control}

To analyze optimal control problems, we adopt the Lagrangian approach [4], in the linear elliptic case. This approach results complementary to the analysis on optimal control, developed by Lions [10], however it allows a systematical mean to define appropriate a posteriori error estimates.

The goal is to minimize a cost functional $J=J(w, u)$ so that a state equation is satisfied, i.e.:

$$
\left\{\begin{array}{l}
J=J(w, u) \quad \text { minimum } \\
A w=f+B u
\end{array}\right.
$$

where $A$ is an elliptic differential operator, $w$ is the state variable, $f$ a source term and $B$ a differential operator acting on the control variable $u$ in state equation. By introducing the Lagrangian functional and a Lagrangian multiplier $p$ :

$$
\mathcal{L}(w, p, u):=J(w, u)+\langle p, f+B u-A w\rangle,
$$

problem (1) has a unique solution (for the linear case see [10]) which satisfies the PDE system:

$$
\nabla \mathcal{L}(w, p, u)=0
$$

Considering $w \in \mathcal{V}, u \in \mathcal{U}$, with $\mathcal{V}$ and $\mathcal{U}$ being two suitable Hilbert spaces, we have the following weak form for state equation (1):

$$
a(w, \varphi)=(f, \varphi)+b(u, \varphi), \quad \forall \varphi \in \mathcal{V},
$$

where $(\cdot, \cdot)$ is the $L^{2}(\Omega)$ inner product, $a(\cdot, \cdot)$ is a bilinear form associated to the linear elliptic operator while $b(\cdot, \cdot)$ is the bilinear form associated with $B u$, that is $b(u, \varphi)=\langle B u, \varphi\rangle$. We assume that the cost functional has the 
following form $[10]$ :

$$
J(w, u):=\frac{1}{2}\left\|C w-z_{d}\right\|^{2}+\frac{1}{2} n(u, u),
$$

where the operator $C$ takes the state variable $w$ into the observation space $\mathcal{Z}$ (a Hilbert space), $z_{d}$ is the observation function, $\|\cdot\|$ is a suitable norm, and $n(\cdot, \cdot)$ is a non-negative Hermitian form. Then:

$$
\mathcal{L}(w, p, u)=J(w, u)+b(u, p)+(f, p)-a(w, p)
$$

The optimal control solution consists in solving the following problem:

$$
\text { find }(w, p, u) \in \mathcal{V} \times \mathcal{V} \times \mathcal{U} \text { s.t. } \nabla \mathcal{L}(w, p, u)[(\phi, \varphi, \psi)]=0, \quad \forall(\phi, \varphi, \psi) \in \mathcal{V} \times \mathcal{V} \times \mathcal{U}
$$

Derivatives are to be intended in Fréchet sense [8], so that, from equation (7), we have:

$$
\left\{\begin{array}{rlrl}
\mathcal{L},,_{w}[\phi] & =\left(C w-z_{d}, C \phi\right)-a(\phi, p)=0, & & \forall \phi \in \mathcal{V}, \\
\mathcal{L},,_{p}[\varphi]=(f, \varphi)+b(u, \varphi)-a(w, \varphi)=0, & & \forall \varphi \in \mathcal{V}, \\
\mathcal{L},_{u}[\psi]=b(\psi, p)+n(u, \psi)=0, & \forall \psi \in \mathcal{U}
\end{array}\right.
$$

The differential $\mathcal{L},_{p}$ corresponds, in weak form, to the state equation, $\mathcal{L},_{w}$ to the adjoint equation and $\mathcal{L}{ }_{u}$ to an optimal control constraint. The function $p$ is the adjoint variable and expresses the cost functional sensitivity to the variations of the control variable $u$; the optimal control constraint $\mathcal{L},{ }_{u}[\psi]$, in weak form, can be related to a strong derivative $J^{\prime}$ (cost functional derivative) by means of Riesz Theorem. Indeed, $\mathcal{L},_{u}[\psi]=\left\langle J^{\prime}(p, u), \psi\right\rangle=\left(J^{\prime}(p, u), \psi\right)$.

The control problem is solved by an iterative method, starting with an initial value for the control variable $u^{0}$. At each step $j \geq 0$ we solve the state equation; then we compute the value of the cost functional and we solve the adjoint equation. Once $p^{j}$ is available, we determine the cost functional derivative $J^{\prime}\left(p^{j}, u^{j}\right)$ and apply a suitable stopping criterium. If this criterium is not fulfilled, we adopt an optimization iteration on control function $u$; for example we use a steepest-descent method:

$$
u^{j+1}=u^{j}-\tau^{j} J^{\prime}\left(p^{j}, u^{j}\right)
$$

where $\tau^{j}$ is a relaxation parameter that can be determined by analyzing the mathematical properties of the control problem $[1,19]$.

\subsection{Optimal control problem governed by an advection-diffusion equation}

We apply the general approach for linear elliptic problems proposed in the previous section to the case of an advection-diffusion state equation:

$$
\left\{\begin{array}{l}
L(w):=-\nabla \cdot(\nu \nabla w)+\mathbf{V} \cdot \nabla w=u, \quad \text { in } \Omega \subset \mathbb{R}^{2}, \\
w=0, \quad \text { on } \Gamma_{D} \\
\nu \frac{\partial w}{\partial n}=0, \quad \text { on } \Gamma_{N},
\end{array}\right.
$$

where $u \in L^{2}(\Omega)$ is the control function defined on the domain $\Omega, w=w(u)$ is the state variable, while $\nu$ and $\mathbf{V}$ depend on the domain coordinates $(x, y)$. We have imposed a homogeneous Dirichlet condition over inflow boundary $\Gamma_{D}:=\{\mathbf{x} \in \partial \Omega: \mathbf{V}(\mathbf{x}) \cdot \mathbf{n}(\mathbf{x})<0\}$, where $\mathbf{n}(\mathbf{x})$ is the unit vector directed outward, and homogeneous Neumann condition on the outflow boundary $\Gamma_{N}:=\partial \Omega \backslash \Gamma_{D}$. Let us notice that, for the sake of simplicity, we have considered homogeneous conditions on both Dirichlet and Neumann boundaries; however our approach is straightforward to apply to problems with non-homogeneous boundary conditions. Defining the observation of the system on a part $D$ of the domain $\Omega(D \subseteq \Omega)$, the optimal control problem reads:

$$
\text { find } u \quad: \quad J(w, u):=\frac{1}{2} \int_{D}\left(g w(u)-z_{d}\right)^{2} \mathrm{~d} D \quad \text { minimum }
$$




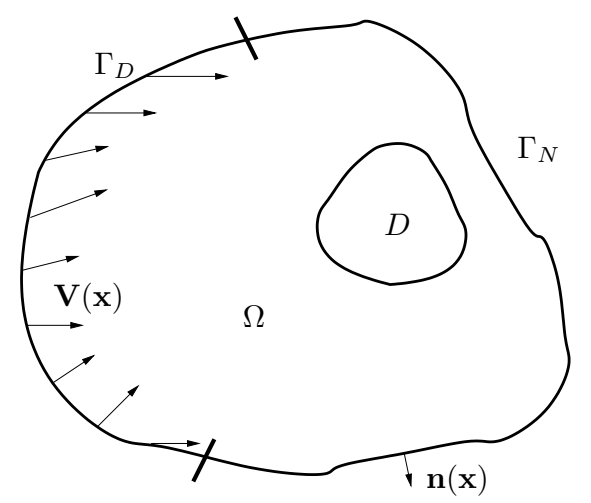

Figure 1. Domain for the advection-diffusion control problem (11).

where $D \subseteq \Omega$ is the domain of observation, $g \in C^{\infty}(D)$ is a function that project the state solution $w$ in the observation space, while $z_{d}$ represents the desired observation function. Note that Equation (11) is a special case of Equation (5) in which $n(\cdot, \cdot)=0$. The domain reported in Figure 1 is typical for this kind of problems.

Defining $H_{\Gamma_{D}}^{1}:=\left\{v \in H^{1}(\Omega): v_{\mid \Gamma_{D}}=0\right\}$, the weak form of Equation (10) reads:

$$
\text { find } w=w(u) \in H_{\Gamma_{D}}^{1}(\Omega): \quad a(w, \varphi)=F(\varphi ; u), \quad \forall \varphi \in H_{\Gamma_{D}}^{1}(\Omega)
$$

where:

$$
\begin{gathered}
a(w, \varphi):=\int_{\Omega} \nu \nabla w \cdot \nabla \varphi \mathrm{d} \Omega+\int_{\Omega} \mathbf{V} \cdot \nabla w \varphi \mathrm{d} \Omega \\
F(\varphi ; u):=\int_{\Omega} u \varphi \mathrm{d} \Omega .
\end{gathered}
$$

The Lagrangian functional becomes:

$$
\mathcal{L}(w, p, u):=J(w, u)+F(p ; u)-a(w, p)
$$

where $p \in H_{\Gamma_{D}}^{1}(\Omega)$ is the Lagrange multiplier. By differentiating $\mathcal{L}$ with respect to the state variable $\left(\mathcal{L},{ }_{w}[\phi]\right)$, we obtain the adjoint equation in weak form:

$$
\text { find } p \in H_{\Gamma_{D}}^{1}(\Omega): \quad a^{a d}(p, \phi)=F^{a d}(\phi ; w), \quad \forall \phi \in H_{\Gamma_{D}}^{1}(\Omega),
$$

where:

$$
\begin{gathered}
a^{a d}(p, \phi):=\int_{\Omega} \nu \nabla p \cdot \nabla \phi \mathrm{d} \Omega+\int_{\Omega} \mathbf{V} \cdot \nabla \phi p \mathrm{~d} \Omega, \\
F^{a d}(\phi ; w)=\int_{D}\left(g w-z_{d}\right) g \phi \mathrm{d} D,
\end{gathered}
$$

that is (in distributional sense):

$$
\left\{\begin{array}{l}
L^{a d}(p):=-\nabla \cdot(\nu \nabla p+\mathbf{V} p)=\chi_{D} g\left(g w-z_{d}\right), \quad \text { in } \Omega \\
p=0, \text { on } \Gamma_{D}, \\
\nu \frac{\partial p}{\partial n}+\mathbf{V} \cdot \mathbf{n} p=0, \quad \text { on } \Gamma_{N}
\end{array}\right.
$$


where $\chi_{D}$ is the characteristic function of the subdomain $D$. By differentiating $\mathcal{L}$ with respect to the control function $u$ we obtain the optimal condition constraint in weak form:

$$
\mathcal{L}_{u}[\psi]=\int_{\Omega} p \psi \mathrm{d} \Omega=0, \quad \forall \psi \in L^{2}(\Omega),
$$

from which we obtain the cost functional derivative:

$$
J^{\prime}(p)=p .
$$

If we use the iterative procedure (9), in the current case the following stopping criterium can be adopted:

$$
\left\|J^{\prime}(p)\right\|_{L^{2}(\Omega)}=\|p\|_{L^{2}(\Omega)}<\text { Tol. }
$$

\section{NumericAl DiscretizATION: THE STABILIZED LAGRANGIAN}

To solve the control problem we adopt an iterative method as described in Section 1.1. At each step, we solve both the state and adjoint equations by a Galerkin finite element method using linear elements on unstructured meshes, composed by triangular elements $\{K\}$ s.t. $\bigcup_{K \in \mathcal{T}_{h}}=\bar{\Omega}$, where $\mathcal{T}_{h}$ is the union of triangles covering the domain $\Omega$.

Both state and adjoint equations are diffusion-transport equations, in which the transport term can dominate the diffusion term; a suitable stabilization is mandatory. To avoid numerical instabilities and their propagation in the course of the optimization iterative method, we propose a stabilization directly on the Lagrangian functional, introducing the stabilized Lagrangian concept, instead of stabilizing separately state and adjoint equations [3] in a conventional manner [16].

The stabilized Lagrangian is defined as:

$$
\mathcal{L}_{h}(w, p, u):=\mathcal{L}(w, p, u)+S_{h}(w, p, u)
$$

with

$$
S_{h}(w, p, u):=\sum_{K \in \mathcal{T}_{h}} \delta_{K} \int_{K} R(w ; u) R^{a d}(p ; w) \mathrm{d} K .
$$

The terms $R(w ; u)$ and $R^{a d}(p ; w)$ represent the residuals of the state and adjoint equations respectively, while $\delta_{K}$ is a stabilization parameter depending on the local Péclet number $\mathbb{P} e_{K}:=\frac{|\mathbf{V}|_{\infty, K} h_{K}}{2 \nu}[7]$, being $h_{K}$ the diameter of the element $K \in \mathcal{T}_{h}$, while $|\mathbf{V}|_{\infty, K}$ is the maximum length of $\mathbf{V}$ on $K$. More precisely, from Equations (10) and (19), we obtain:

$$
\begin{gathered}
R(w ; u):=L(w)-u, \\
R^{a d}(p ; w):=L^{a d}(p)-G(w), \\
G(w):=\chi_{D} g\left(g w-z_{d}\right) .
\end{gathered}
$$

Considering the Galerkin-FE approximation and differentiating the stabilized Lagrangian with respect to the adjoint variable, we obtain the stabilized state equation in weak form:

$$
\text { find } w_{h} \in X_{h}: \quad a\left(w_{h}, \varphi_{h}\right)+s_{h}\left(w_{h}, \varphi_{h} ; u_{h}\right)=F\left(\varphi_{h} ; u_{h}\right), \quad \forall \varphi_{h} \in X_{h},
$$

with:

$$
s_{h}\left(w_{h}, \varphi_{h} ; u_{h}\right):=-\sum_{K \in \mathcal{T}_{h}} \delta_{K} \int_{K} R\left(w_{h} ; u_{h}\right) L^{a d}\left(\varphi_{h}\right) \mathrm{d} K .
$$

Equivalently, the state equation reads:

$$
\text { find } w_{h} \in X_{h}: \quad a_{h}\left(w_{h}, \varphi_{h}\right)=F_{h}\left(\varphi_{h} ; u_{h}\right), \quad \forall \varphi_{h} \in X_{h},
$$


where:

$$
\begin{gathered}
a_{h}\left(w_{h}, \varphi_{h}\right):=a\left(w_{h}, \varphi_{h}\right)-\sum_{K \in \mathcal{T}_{h}} \delta_{K} \int_{K} L\left(w_{h}\right) L^{a d}\left(\varphi_{h}\right) \mathrm{d} K, \\
F_{h}\left(\varphi_{h} ; u_{h}\right):=F\left(\varphi_{h} ; u_{h}\right)-\sum_{K \in \mathcal{T}_{h}} \delta_{K} \int_{K} u_{h} L^{a d}\left(\varphi_{h}\right) \mathrm{d} K .
\end{gathered}
$$

Similarly, by differentiating the stabilized Lagrangian with respect to the state variable, we obtain the stabilized adjoint equation:

$$
\text { find } p_{h} \in X_{h}: \quad a^{a d}\left(p_{h}, \phi_{h}\right)+s_{h}^{a d}\left(p_{h}, \phi_{h} ; w_{h}\right)=F^{a d}\left(\phi_{h} ; w_{h}\right), \quad \forall \phi_{h} \in X_{h},
$$

where:

$$
s_{h}^{a d}\left(p_{h}, \phi_{h} ; w_{h}\right):=-\sum_{K \in \mathcal{T}_{h}} \delta_{K} \int_{K}\left(R^{a d}\left(p_{h} ; w_{h}\right) L\left(\phi_{h}\right)-R\left(w_{h} ; u_{h}\right) G^{\prime}\left(\phi_{h}\right)\right) \mathrm{d} K,
$$

and:

$$
G^{\prime}(w):=\chi_{D} g^{2} w
$$

Equivalently, the adjoint equation reads:

$$
\text { find } p_{h} \in X_{h}: a_{h}^{a d}\left(p_{h}, \phi_{h}\right)=F_{h}^{a d}\left(\phi_{h} ; w_{h} ; u_{h}\right), \quad \forall \phi_{h} \in X_{h},
$$

where:

$$
a_{h}^{a d}\left(p_{h}, \phi_{h}\right):=a^{a d}\left(p_{h}, \phi_{h}\right)-\sum_{K \in \mathcal{T}_{h}} \delta_{K} \int_{K} L^{a d}\left(p_{h}\right) L\left(\phi_{h}\right) \mathrm{d} K
$$

and:

$$
F_{h}^{a d}\left(\phi_{h} ; w_{h} ; u_{h}\right):=F^{a d}\left(\phi_{h} ; w_{h}\right)-\sum_{K \in \mathcal{T}_{h}} \delta_{K} \int_{K}\left(G\left(w_{h}\right) L\left(\phi_{h}\right)+R\left(w_{h} ; u_{h}\right) G^{\prime}\left(\phi_{h}\right)\right) \mathrm{d} K .
$$

Notice that the terms $a\left(w_{h}, \varphi_{h}\right), F\left(\varphi_{h} ; u_{h}\right), a^{a d}\left(p_{h}, \phi_{h}\right)$ and $F^{a d}\left(\phi_{h} ; w_{h}\right)$ are defined in Equations (13), (14), (17) and (18), $u_{h}$ is a discrete approximation of the control function $u$ and $X_{h} \subset H_{\Gamma_{D}}^{1}$ is the finite element space. In this way we obtain discrete state and adjoint equations that can be regarded as Galerkin approximations to the exact state and adjoint equations, plus extra terms deriving from differentiating the term $S_{h}$ in Equation (23). Considering the state equation, we note that this extra term differs from the one that we could have obtained by stabilizing directly the state equation by means of a strongly consistent method like GLS (Galerkin least squares) [16], that is:

$$
\text { find } w_{h} \in X_{h}: \quad a\left(w_{h}, \varphi_{h}\right)+\tilde{s}_{h}\left(w_{h}, \varphi_{h} ; u_{h}\right)=F\left(\varphi_{h} ; u_{h}\right), \quad \forall \varphi_{h} \in X_{h},
$$

with

$$
\tilde{s}_{h}\left(w_{h}, \varphi_{h} ; u_{h}\right):=\sum_{K \in \mathcal{T}_{h}} \delta_{K} \int_{K} R\left(w_{h} ; u_{h}\right) L\left(\varphi_{h}\right) \mathrm{d} K .
$$

In the same manner we can apply the stabilization by a GLS method to the adjoint equation, obtaining the following weak form:

$$
\text { find } p_{h} \in X_{h}: \quad a^{a d}\left(p_{h}, \phi_{h}\right)+\tilde{s}_{h}^{a d}\left(p_{h}, \phi_{h} ; w_{h}\right)=F^{a d}\left(\phi_{h} ; w_{h}\right), \quad \forall \phi_{h} \in X_{h},
$$

with

$$
\tilde{s}_{h}^{a d}\left(p_{h}, \phi_{h} ; w_{h}\right):=\sum_{K \in 2 \mathcal{T}_{h}} \delta_{K} \int_{K} R^{a d}\left(p_{h} ; w_{h}\right) L^{a d}\left(\phi_{h}\right) \mathrm{d} K
$$


Note that Equation (41) is not the adjoint of Equation (39). On the contrary, with the approach that we advocate in this paper, Equation (36) is indeed the adjoint of Equation (30), that is $a_{h}\left(w_{h}, p_{h}\right)=a_{h}^{a d}\left(p_{h}, w_{h}\right)$ for all $w_{h}$ and $p_{h}$, as it can be grasped from a direct inspection of Equations (31) and (37).

Finally, differentiating $\mathcal{L}_{h}$ with respect to $u_{h}$, we obtain:

$$
J^{\prime}\left(p_{h}, w_{h}\right)=p_{h}-\sum_{K \in \mathcal{T}_{h}} \delta_{K} R^{a d}\left(p_{h} ; w_{h}\right) ;
$$

the associated stopping criterium reads:

$$
\left\|J^{\prime}\left(p_{h}, w_{h}\right)\right\|_{L^{2}(\Omega)}=\left\|p_{h}-\sum_{K \in \mathcal{T}_{h}} \delta_{K} R^{a d}\left(p_{h} ; w_{h}\right)\right\|_{L^{2}(\Omega)}<T o l .
$$

Equation (43) differs from that achievable from the separate stabilization of the state and adjoint equations, which does not involve the constraint equation and would read: $J^{\prime}\left(p_{h}\right)=p_{h}$.

This approach features the following properties:

- stabilization is based on a strongly consistent method [16] (this will allow a convenient a posteriori error estimates in the field of estimates with residuals);

- there is coherence between state and adjoint stabilized equations;

- as we will see in Section 3.3, this is a systematical method to achieve error estimates for linear control problems or functionals depending on PDE solution [3], without introducing second order terms (that need to be bounded).

Remark 2.1. For the sake of simplicity in this work we have adopted an advection-diffusion state equation (see Eq. (10)); however the control problem and the stabilized Lagrangian concept can be applied in the same manner for an equation that contains a reaction (zero-th order) term.

\section{A POSTERIORI ERROR ESTIMATE}

To obtain appropriate a posteriori error estimates for our optimal control problem, we consider a strategy dealing with error on cost functional [4], rather than the sum of errors on state, adjoint and control variables $[9,11,12]$. In fact, the error on cost functional can be bounded by suitable combination of the errors on state, adjoint and control variables, as we shall see in Section 3.3.

\subsection{Separating iteration error from discretization error}

At the iterative step $j$ the adaptivity will be based on the evaluation of the following error [4]:

$$
\left|J\left(w^{*}, u^{*}\right)-J\left(w_{h}^{j}, u_{h}^{j}\right)\right|,
$$

where $*$ indicates optimal variables. Equation (45) involves both the iteration and discretization errors, say $\varepsilon_{I T}^{(j)}$ and $\varepsilon_{D}^{(j)}$, since:

$$
J\left(w^{*}, u^{*}\right)-J\left(w_{h}^{j}, u_{h}^{j}\right)=\underbrace{J\left(w^{*}, u^{*}\right)-J\left(w^{j}, u^{j}\right)}_{\varepsilon_{I T}^{(j)}}+\underbrace{J\left(w^{j}, u^{j}\right)-J\left(w_{h}^{j}, u_{h}^{j}\right)}_{\varepsilon_{D}^{(j)}},
$$

the iteration error being the distance between the cost functional ideally computed on continuous variables at the step $j$ and the cost functional on the optimum. If we use an adaptive procedure based on grid refinement, to reduce error (45), we make sure to reduce the component due to the discretization error, but not that of the iteration error. Indeed, although the latter is grid independent, when evaluated on the new grid, generally it increases, so that, after grid adaptation, error (45) can get larger. To avoid this problem we propose an adaptivity 


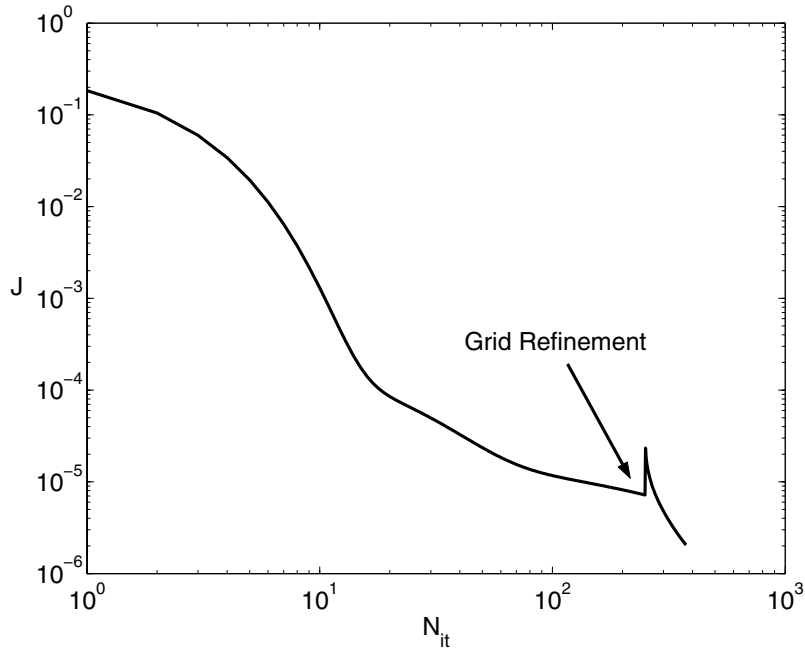

(a)

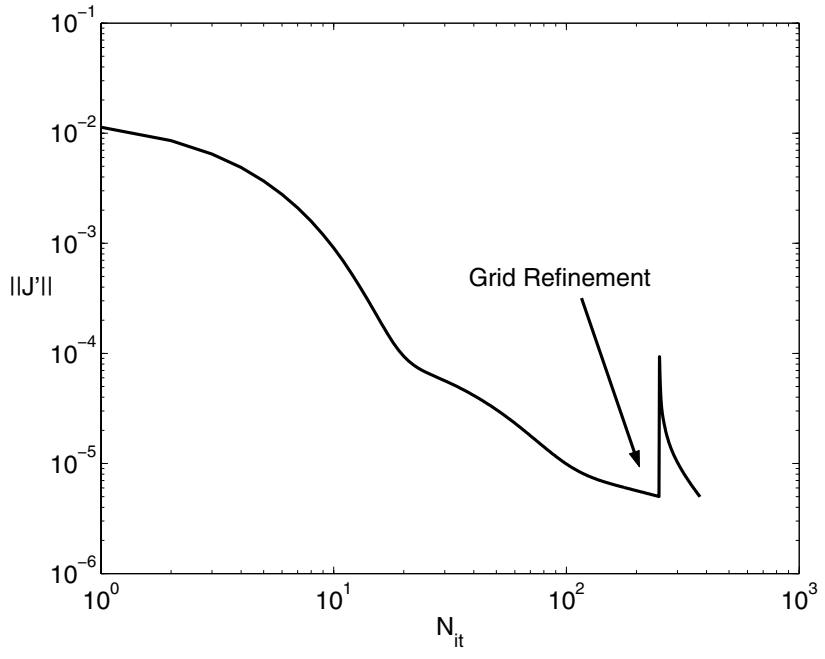

(b)

Figure 2. Convergence history for cost functional $J$ (a) and iteration error (evaluated as $\left\|J^{\prime}\right\|$ ) (b) (in $\log -\log$ scale) for problem (47) with $w_{d}=5 y(1-y) \sin (2 \pi x)$; the effect of grid refinement is outlined.

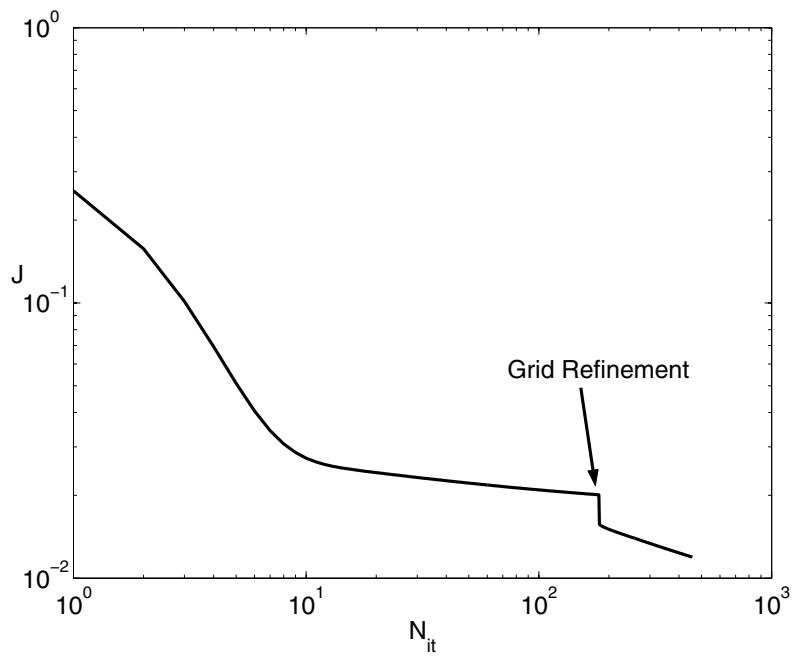

(a)

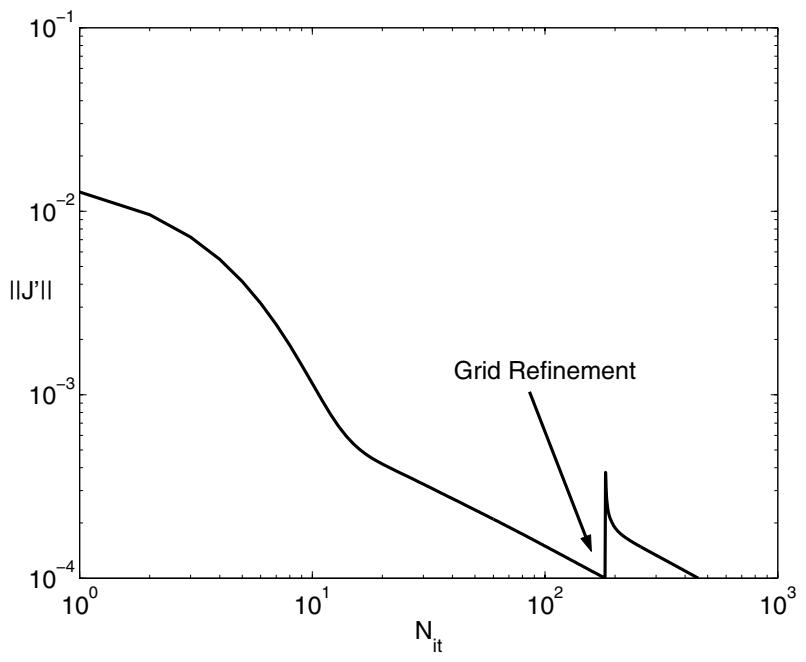

(b)

Figure 3. Convergence history for cost functional $J$ (a) and iteration error (evaluated as $\left.\left\|J^{\prime}\right\|\right)$ (b) (in $\log -\log$ scale) for problem (47) with $w_{d}=5 y(1-y) \sin (2 \pi x)+e^{-10 x}$; the effect of grid refinement is outlined.

based, at each step, on the evaluation of the discretization error $\left|J\left(w^{j}, u^{j}\right)-J\left(w_{h}^{j}, u_{h}^{j}\right)\right|$; this approach is effective since the very early stages of the optimization procedure, when we are still far from the optimal solution.

Figures 2 and 3 are instances of the effect of grid refinement on the behavior of the cost functional $J\left(w_{h}, u_{h}\right)$ and the iteration error along the iterations of the optimization method. More specifically, we refer to the 
following problem:

find $u: J(w, u)=\frac{1}{2} \int_{\Omega}\left(w-w_{d}\right)^{2} \mathrm{~d} \Omega$ minimum, $\quad$ with $\quad \begin{cases}-\Delta w=u, & \text { in } \Omega=(0,1) \times(0,1), \\ w=0, & \text { on } \partial \Omega,\end{cases}$

where we solve the problem by means of the optimization method proposed in Section 1.1, starting by $u^{0}=0$ and assuming $\tau=700$. We solve problem by piecewise linear finite elements, initially on a coarse grid of 130 elements, then on a uniformly refined grid with 2080 elements. In one case (Fig. 2), for which $w_{d}=5 y(1-y) \sin (2 \pi x)$ and stopping criterion $\left\|J^{\prime}\right\|<T o l=5 \times 10^{-6}, J$ is increasing with grid refinement, while in the other (Fig. 3), with $w_{d}=5 y(1-y) \sin (2 \pi x)+\mathrm{e}^{-10 x}$ and stopping criterion $\left\|J^{\prime}\right\|<T o l=10^{-4}, J$ is decreasing when re-evaluated on the fine grid. Notice that we identify the iteration error with the cost functional derivative $J^{\prime}\left(p_{h}, u_{h}\right)$ (in an appropriate norm), as we will see in Section 3.2; in particular we plot the iteration error re-evaluated on the fine grid.

\subsection{Evaluation of the iteration error}

Definition 3.1. A control problem is linear if $\nabla \mathcal{L}(\mathbf{x})$ is linear in $\mathbf{x}:=(w, p, u)$.

This is the case, e.g., if the control problem is governed by a linear state equation and the cost functional is quadratic with respect to both $w$ and $u$.

Theorem 3.2. For linear control problems, the iteration error at the $j$-th iteration has the following expression:

$$
\varepsilon_{I T}^{(j)}=\frac{1}{2}\left(J^{\prime}\left(p^{j}, u^{j}\right), u^{*}-u^{j}\right)
$$

Proof. We have $\varepsilon_{I T}^{(j)}=\mathcal{L}\left(\mathbf{x}^{*}\right)-\mathcal{L}\left(\mathbf{x}^{j}\right)$, where $\mathbf{x}^{*}:=\left(w^{*}, p^{*}, u^{*}\right)$ and $\mathbf{x}^{j}:=\left(w^{j}, p^{j}, u^{j}\right)$, being the state equation satisfied adopting continuous variables. From the Fundamental Calculus Theorem, we obtain:

$$
\varepsilon_{I T}^{(j)}=\int_{\mathbf{x}^{j}}^{\mathbf{x}^{*}} \nabla \mathcal{L}(\overline{\mathbf{x}}) \cdot \overline{\mathbf{x}}=\int_{0}^{1} \nabla \mathcal{L}\left(\mathbf{x}^{j}+s\left(\mathbf{x}^{*}-\mathbf{x}^{j}\right)\right) \cdot\left(\mathbf{x}^{*}-\mathbf{x}^{j}\right) \mathrm{d} s,
$$

being $\overline{\mathbf{x}}:=(\bar{w}, \bar{p}, \bar{u})$ and having applied the variables transformation $\overline{\mathbf{x}}=\mathbf{x}^{j}+s\left(\mathbf{x}^{*}-\mathbf{x}^{j}\right)$, with $s \in \mathbb{R}$. By writing [14]:

$$
\varepsilon_{I T}^{(j)}=\nabla \mathcal{L}\left(\mathbf{x}^{j}\right) \cdot\left(\mathbf{x}^{*}-\mathbf{x}^{j}\right)+\int_{0}^{1}\left(\nabla \mathcal{L}\left(s \mathbf{x}^{*}+(1-s) \mathbf{x}^{j}\right)-\nabla \mathcal{L}\left(\mathbf{x}^{j}\right)\right) \cdot\left(\mathbf{x}^{*}-\mathbf{x}^{j}\right) \mathrm{d} s,
$$

and using the fact that $\nabla \mathcal{L}(\mathbf{x})$ is linear and the optimal condition $\nabla \mathcal{L}\left(\mathbf{x}^{*}\right)=0$, we obtain [14]

$$
\varepsilon_{I T}^{(j)}=\nabla \mathcal{L}\left(\mathbf{x}^{j}\right) \cdot\left(\mathbf{x}^{*}-\mathbf{x}^{j}\right)+\int_{0}^{1} s \nabla \mathcal{L}\left(\mathbf{x}^{*}-\mathbf{x}^{j}\right) \cdot\left(\mathbf{x}^{*}-\mathbf{x}^{j}\right) d s=\frac{1}{2} \nabla \mathcal{L}\left(\mathbf{x}^{j}\right) \cdot\left(\mathbf{x}^{*}-\mathbf{x}^{j}\right) .
$$

Being $w^{j}$ and $p^{j}$ the exact state and adjoint solutions respectively, we finally have:

$$
\varepsilon_{I T}^{(j)}=\frac{1}{2} \mathcal{L}_{, u}\left(\mathbf{x}^{j}\right)\left[u^{*}-u^{j}\right]=\frac{1}{2}\left(J^{\prime}\left(p^{j}, u^{j}\right), u^{*}-u^{j}\right) .
$$

Corollary 3.3. If a steepest-descent iterative method with constant relaxation parameter $\tau$ is used, the iteration error can be written as:

$$
\varepsilon_{I T}^{(j)}=-\frac{1}{2} \tau\left\|J^{\prime}\left(p^{j}, u^{j}\right)\right\|^{2}-\frac{1}{2} \tau \sum_{r=j+1}^{\infty}\left(J^{\prime}\left(p^{j}, u^{j}\right), J^{\prime}\left(p^{r}, u^{r}\right)\right) .
$$


Proof. In Equation (48) we replace $u^{*}-u^{j}$ with $u^{*}-u^{j+1}-\tau J^{\prime}\left(p^{j}, u^{j}\right)$ (see Eq. (9)). By proceeding recursively we obtain:

$$
\varepsilon_{I T}^{(j)}=\frac{1}{2}\left(J^{\prime}\left(p^{j}, u^{j}\right), \sum_{r=j}^{\infty}-\tau J^{\prime}\left(p^{r}, u^{r}\right)\right),
$$

from which the result follows.

By applying Equation (49) to the advection-diffusion control problem defined in Section 1.2, we obtain:

$$
\varepsilon_{I T}^{(j)}=-\frac{1}{2} \tau\left\|p^{j}\right\|_{L^{2}(\Omega)}^{2}-\frac{1}{2} \tau \sum_{r=j+1}^{\infty}\left(p^{j}, p^{r}\right)_{L^{2}(\Omega)}
$$

Notice that we can not evaluate correctly this error, since we don't know the values of $p^{r}$ for $r \geq j+1$. For this reason we can estimate the iteration error as $\left|\varepsilon_{I T}^{(j)}\right| \approx \frac{1}{2} \tau\left\|p^{j}\right\|_{L^{2}(\Omega)}^{2}$, or more simply:

$$
\left|\varepsilon_{I T}^{(j)}\right| \approx\left\|p^{j}\right\|_{L^{2}(\Omega)}^{2},
$$

that leads to the usual stopping criterium: $\left\|J^{\prime}\left(p^{j}\right)\right\|<$ Tol.

\subsection{A posteriori estimate for the discretization error}

We want to estimate the discretization error in Equation (47).

Theorem 3.4. For a linear control problem with a strongly consistent stabilized Lagrangian $\mathcal{L}_{h}$ (Eqs. (23) and (24)), letting $\mathbf{x}_{h}^{j}:=\left(w_{h}^{j}, p_{h}^{j}, u_{h}^{j}\right)$ be the Galerkin approximation, the discretization error at the $j$-th iteration becomes:

$$
\varepsilon_{D}^{(j)}=\frac{1}{2}\left(J^{\prime}\left(p^{j}, u^{j}\right), u^{j}-u_{h}^{j}\right)+\frac{1}{2} \nabla \mathcal{L}_{h}\left(\mathbf{x}_{h}^{j}\right) \cdot\left(\mathbf{x}^{j}-\mathbf{x}_{h}^{j}\right)+\Lambda_{h}\left(\mathbf{x}_{h}^{j}\right),
$$

where $\Lambda_{h}\left(\mathbf{x}_{h}^{j}\right):=S_{h}\left(\mathbf{x}_{h}^{j}\right)+s_{h}\left(w_{h}^{j}, p_{h}^{j} ; u_{h}^{j}\right)$, being $s_{h}\left(w_{h}^{j}, p_{h}^{j} ; u_{h}^{j}\right)$ the stabilization term (Eq. (29)) induced from Equation (23) on the state equation (Eq. (28)).

Proof. Taking into account Equation (15), we have:

$$
J\left(w^{j}, u^{j}\right)=\mathcal{L}\left(\mathbf{x}^{j}\right)-F\left(p^{j} ; u^{j}\right)+a\left(w^{j}, p^{j}\right) ;
$$

noting that the variables $w^{j}$ and $p^{j}$ are the continuous solutions of the state and adjoint equations corresponding to $u^{j}$, we obtain $J\left(w^{j}, u^{j}\right)=\mathcal{L}\left(\mathbf{x}^{j}\right)$. In the same manner, from Equations (15) and (23), we can express $J\left(w_{h}^{j}, u_{h}^{j}\right)$ as:

$$
J\left(w_{h}^{j}, u_{h}^{j}\right)=\mathcal{L}_{h}\left(\mathbf{x}_{h}^{j}\right)-S_{h}\left(\mathbf{x}_{h}^{j}\right)-F\left(p_{h}^{j} ; u_{h}^{j}\right)+a\left(w_{h}^{j}, p_{h}^{j}\right) .
$$

Adding and subtracting the term $s_{h}\left(w_{h}^{j}, p_{h}^{j} ; u_{h}^{j}\right)$ to the right hand side of the previous equation, we obtain that $J\left(w_{h}^{j}, u_{h}^{j}\right)=\mathcal{L}_{h}\left(\mathbf{x}_{h}^{j}\right)-\Lambda_{h}\left(\mathbf{x}_{h}^{j}\right)$, since $w_{h}^{j}$ satisfies a state equation like Equation (28) (with a right hand side depending on $j$ ) in which $p_{h}^{j}$ can play the role of test function.

In order to provide an appropriate estimate, we need to separate entirely the discretization error from the stabilization error:

$$
\varepsilon_{D}^{(j)}=\varepsilon_{D 1}^{(j)}+\varepsilon_{D 2}^{(j)}+\Lambda_{h}\left(\mathbf{x}_{h}^{j}\right)=\left(\mathcal{L}\left(\mathbf{x}^{j}\right)-\mathcal{L}_{h}\left(\mathbf{x}^{j}\right)\right)+\left(\mathcal{L}_{h}\left(\mathbf{x}^{j}\right)-\mathcal{L}_{h}\left(\mathbf{x}_{h}^{j}\right)\right)+\Lambda_{h}\left(\mathbf{x}_{h}^{j}\right) .
$$

The expression of $\varepsilon_{D 1}^{(j)}$ is available only if an expression for $\mathcal{L}_{h}$ is known, as in the case of the stabilized Lagrangian (Eq. (23)); this would not be the case when the state and adjoint equations are stabilized directly [3]. From 
Equations (23) and (24), we have:

$$
\varepsilon_{D 1}^{(j)}=-S_{h}\left(w^{j}, p^{j}, u^{j}\right)=-\sum_{K \in \mathcal{T}_{h}} \delta_{K}\left(R\left(w^{j} ; u^{j}\right), R^{a d}\left(p^{j} ; w^{j}\right)\right) .
$$

This term is identically zero, due to the property of strong consistency. By applying the fundamental calculus Theorem, using the linearity of $\nabla \mathcal{L}_{h}(\mathbf{x})[14]$, observing that $\nabla \mathcal{L}\left(\mathbf{x}^{j}\right) \cdot\left(\mathbf{x}^{j}-\mathbf{x}_{h}^{j}\right) \equiv \mathcal{L}_{, u}\left(\mathbf{x}^{j}\right)\left[u^{j}-u_{h}^{j}\right]$ for the continuous variables and proceeding as done in Theorem 3.2, we obtain:

$$
\varepsilon_{D 2}^{(j)}=\frac{1}{2}\left(J^{\prime}\left(p^{j}, u^{j}\right), u^{j}-u_{h}^{j}\right)+\frac{1}{2} \nabla \mathcal{L}_{h}\left(\mathbf{x}_{h}^{j}\right) \cdot\left(\mathbf{x}^{j}-\mathbf{x}_{h}^{j}\right)
$$

Then Equation (52) follows.

Now we apply the abstract result of Theorem 3.4 to the advection-diffusion control problem defined in Section 1.2. From Equations (24) and (29) we have:

$$
\Lambda_{h}\left(\mathbf{x}_{h}^{j}\right)=-\sum_{K \in \mathcal{T}_{h}} \delta_{K} \int_{K} G\left(w_{h}^{j}\right) R\left(w_{h}^{j} ; u_{h}^{j}\right) \mathrm{d} K
$$

Putting into evidence the contributions of the single elements $K \in \mathcal{T}_{h}$ (see e.g. [3,4]), we obtain:

$$
\left|\varepsilon_{D}^{(j)}\right| \leq \eta_{D}^{(j)}:=\frac{1}{2} \sum_{K \in \mathcal{T}_{h}}\left\{\left(\omega_{K}^{p} \rho_{K}^{w}+\omega_{K}^{w} \rho_{K}^{p}+\omega_{K}^{u} \rho_{K}^{u}\right)+\lambda_{K}\right\}
$$

where (see Sect. 2 for symbol definitions):

$$
\begin{aligned}
& \rho_{K}^{w}:=\left\|R\left(w_{h}^{j} ; u_{h}^{j}\right)\right\|_{K}+h_{K}^{-\frac{1}{2}}\left\|r\left(w_{h}^{j}\right)\right\|_{\partial K}, \\
& \omega_{K}^{p}:=\left\|\left(p^{j}-p_{h}^{j}\right)-\delta_{K} L^{a d}\left(p^{j}-p_{h}^{j}\right)+\delta_{K} G^{\prime}\left(w^{j}-w_{h}^{j}\right)\right\|_{K}+h_{K}^{\frac{1}{2}}\left\|p^{j}-p_{h}^{j}\right\|_{\partial K}, \\
& \rho_{K}^{p}:=\left\|R^{a d}\left(p_{h}^{j} ; w_{h}^{j}\right)\right\|_{K}+h_{K}^{-\frac{1}{2}}\left\|r^{a d}\left(p_{h}^{j}\right)\right\|_{\partial K}, \\
& \omega_{K}^{w}:=\left\|\left(w^{j}-w_{h}^{j}\right)-\delta_{K} L\left(w^{j}-w_{h}^{j}\right)\right\|_{K}+h_{K}^{\frac{1}{2}}\left\|w^{j}-w_{h}^{j}\right\|_{\partial K}, \\
& \rho_{K}^{u}:=\left\|J^{\prime}\left(p_{h}^{j}, w_{h}^{j}\right)+J^{\prime}\left(p^{j}\right)\right\|_{K}=\left\|p^{j}+p_{h}^{j}-\delta_{K} R^{a d}\left(p_{h}^{j} ; w_{h}^{j}\right)\right\|_{K}, \\
& \omega_{K}^{u}:=\left\|u^{j}-u_{h}^{j}\right\|_{K} \text {, } \\
& \lambda_{K}:=2 \delta_{K}\left\|R\left(w_{h}^{j} ; u_{h}^{j}\right)\right\|_{K}\left\|G\left(w_{h}^{j}\right)\right\|_{K}, \\
& r\left(w_{h}^{j}\right):= \begin{cases}-\frac{1}{2}\left[\nu \frac{\partial w_{h}^{j}}{\partial n}\right], & \text { on } \partial K \backslash \partial \Omega, \\
-\nu \frac{\partial w_{h}^{j}}{\partial n}, & \text { on } \partial K \in \Gamma_{N},\end{cases} \\
& r^{a d}\left(p_{h}^{j}\right):= \begin{cases}-\frac{1}{2}\left[\nu \frac{\partial p_{h}^{j}}{\partial n}+\mathbf{V} \cdot \mathbf{n} p_{h}^{j}\right], & \text { on } \partial K \backslash \partial \Omega, \\
-\left(\nu \frac{\partial p_{h}^{j}}{\partial n}+\mathbf{V} \cdot \mathbf{n} p_{h}^{j}\right), & \text { on } \partial K \in \Gamma_{N} .\end{cases}
\end{aligned}
$$

Notice that $\partial K$ indicates the boundary of the element $K \in \mathcal{T}_{h}$, while the term [.] denotes the jump across the single edge $\partial K$ of the embraced quantity. 


\subsubsection{Evaluation of the estimate (54) by quadratic reconstruction}

Before using the estimate (54) we need to evaluate $w^{j}, p^{j}$ and $u^{j}$; to this aim we propose a technique based on quadratic reconstruction of $\mathrm{FE}$-linear solutions:

- $w^{j}$ is replaced by $\left(w_{h}^{j}\right)^{q}$, the quadratic reconstruction of $w_{h}^{j}$;

- $p^{j}$ is replaced by $\left(p_{h}^{j}\right)^{q}$, the quadratic reconstruction of $p_{h}^{j}$;

- when considering the optimization steepest-descent method (with $\tau^{j}=\tau$ constant), $u^{j}$ is replaced by $\left(u_{h}^{j}\right)^{q}:=u_{h}^{j}-\tau\left(J^{\prime}\left(\left(p_{h}^{j}\right)^{q},\left(w_{h}^{j}\right)^{q}\right)-J^{\prime}\left(p_{h}^{j}, w_{h}^{j}\right)\right.$.

We propose the following quadratic reconstruction method, that can be applied for a generic PDE solution $v$ :

- compute the generic numerical solution $v_{h}$ by linear finite elements on the triangular grid $\mathcal{T}_{h}$;

- refine uniformly the mesh $\mathcal{T}_{h}$, dividing each element in four new elements, to obtain the grid $\mathcal{T}_{h}^{f}$;

- resolve the PDE on the fine grid $\mathcal{T}_{h}^{f}$, obtaining the new FE-linear solution $v_{h}^{f}$;

- $\forall K \in \mathcal{T}_{h}$ take the restriction $\left.v_{h}^{f}\right|_{K}$ of $v_{h}^{f}$ to every one of the four elements of $\mathcal{T}_{h}^{f}$ contained in $K$;

- $\forall K \in \mathcal{T}_{h}$ let $v_{h}^{q}$ be the quadratic reconstruction of $\left.v_{h}^{f}\right|_{K}$ (a second order polynomial function on $K$ ), so that the following condition is satisfied[20]:

$$
\text { find } v_{K}^{q} \in \mathbb{P}^{2}(K):\left\|v_{K}^{q}-v_{K}^{f}\right\|_{H^{1}(K)} \text { minimum on } K \text {. }
$$

\subsubsection{Adaptive strategy}

We propose a general strategy to apply to linear control problems:

- we use the optimization iterative method till convergence to iteration error tolerance;

- we adapt the grid with an error balancing criterium over grid elements in an iterative setting led by the error indicator $\eta_{D}^{(j)}(54)$, till convergence to discretization error tolerance;

- we re-evaluate the variables and the iteration error on the adapted grid;

- if the iteration error is increased (as it happens quite frequently), we perform the optimization iterative process once time, till new convergence to prescribed tolerance;

- we re-evaluate the discretization error and, if necessary, we adapt the grid anew;

- we repeat this procedure till when both iteration and discretization errors are below the given tolerances.

This procedure is very convenient computationally: owing to the uniqueness of the solution of linear control problems [10], we can apply the iterative procedure to PDE solved on a coarse grid, and, only near optimal solution, perform mesh adaptation.

In practice two different tolerances for iteration and discretization errors can be adopted; a better strategy should account for error balancing [5], however this might not be convenient if the estimate of the iteration error is too rough.

\section{NUMERICAL TESTS}

In this section we report some numerical tests proving the effectiveness of the proposed methods. Particularly, we refer to two practical problems, that can be interpreted as pollution control problems. The previous one refers to a water pollution situation in which we regulate the emission sources to keep the pollutant concentration below a desired threshold in a river. The second problem deals with an air pollution situation, referring to emissions by industrial plants or chimneys.

To this aim, let us consider the particular case in which the control term $u$ in the state equation (10) is defined only in some regions $U_{i} \subset \Omega$, with $i=1, \ldots, N$. This is the case, e.g. if we have some pollutant sources located in the domain $\Omega$ and we want to regulate them in order to keep the pollutant concentration below a desired threshold in the observation area $D$. In particular we assume $u=\sum_{i=1}^{N} u_{i} \chi_{U_{i}}$, being $\chi_{U_{i}}$ the characteristic function of the subdomain $U_{i}$ and $u_{i}$ the control term located in $U_{i}$, extended by zero outside $U_{i}$. 
According to the choice of $u$, Equation (14) reads:

$$
F(\varphi ; u)=\sum_{i=1}^{N} \int_{U_{i}} u_{i} \varphi \mathrm{d} U_{i}
$$

in the same manner the optimal control constraint (20) is:

$$
\mathcal{L}_{,_{u}}[\psi]=\sum_{i=1}^{N} \int_{U_{i}} p \psi \mathrm{d} U_{i}=0, \quad \forall \psi \in L^{2}(\Omega),
$$

while the cost functional derivative becomes:

$$
J^{\prime}(p)=\sum_{i=1}^{N} p \chi_{U_{i}}=p \sum_{i=1}^{N} \chi_{U_{i}}
$$

which leads to the following stopping criterium: $\left\|J^{\prime}(p)\right\|_{L^{2}(\Omega)}=\left\{\sum_{i}^{N}\|p\|_{L^{2}\left(U_{i}\right)}^{2}\right\}^{\frac{1}{2}}<T o l$.

Applying the new form for $u$ to the discrete problem (see Sect. 2), the cost functional derivative can be expressed as:

$$
J^{\prime}\left(p_{h}, u_{h}\right)=\sum_{i=1}^{N}\left(p_{h}-\sum_{K \in \mathcal{T}_{h}^{i}} \delta_{K} R^{a d}\left(p_{h} ; w_{h}\right)\right) \chi_{U_{i}}
$$

where $\mathcal{T}_{h}^{i}$ is the union of the triangles $\mathrm{K}$ covering $U_{i}$; the associated stopping criterium reads:

$$
\left\|J^{\prime}\left(p_{h}, u_{h}\right)\right\|_{L^{2}(\Omega)}=\left\{\sum_{i=1}^{N}\left\|p_{h}-\sum_{K \in \mathcal{T}_{h}^{i}} \delta_{K} R^{a d}\left(p_{h} ; w_{h}\right)\right\|_{L^{2}\left(U_{i}\right)}^{2}\right\}^{\frac{1}{2}}<\text { Tol } .
$$

Similarly we can adapt to this specific contest the results and the numerical methods proposed in Section 3 . In particular the estimate (54) yields, Equation (55) holds, and in our case the terms $\rho_{K}^{u}$ and $\omega_{K}^{u}$ take the following form:

$$
\begin{aligned}
& \rho_{K}^{u}=\left\|p^{j}+p_{h}^{j}-\delta_{K} R^{a d}\left(p_{h}^{j} ; w_{h}^{j}\right)\right\|_{K \in \bigcup_{i} U_{i}}, \\
& \omega_{K}^{u}=\left\|u^{j}-u_{h}^{j}\right\|_{K \in \bigcup_{i} U_{i}} .
\end{aligned}
$$

We solve numerically both the state and adjoint equations by the Galerkin-FE linear method on an initial (coarse) grid composed by triangular elements. The steepest-descent iterative method is used to solve the control problem, for which we assume a relaxation parameter $\tau$ constant in the iterative process. The grid adaptivity procedure is carried out using the estimate (54) and the method proposed in Section 3; we will compare the results provided by the error indicator $\eta_{D}$ (see Eq. (54)) with those obtained by virtue of the following ones:

- the energy norm error indicator [21] for the state equation, i.e.:

$$
\eta_{E}^{w}:=\sum_{K \in \mathcal{T}_{h}} h_{K} \rho_{K}^{w}
$$

- the error indicator [3]:

$$
\eta_{E}^{w p u}:=\sum_{K \in \mathcal{T}_{h}} h_{K}\left\{\left(\rho_{K}^{w}\right)^{2}+\left(\rho_{K}^{p}\right)^{2}+\left(\rho_{K}^{u}\right)^{2}\right\}^{\frac{1}{2}},
$$




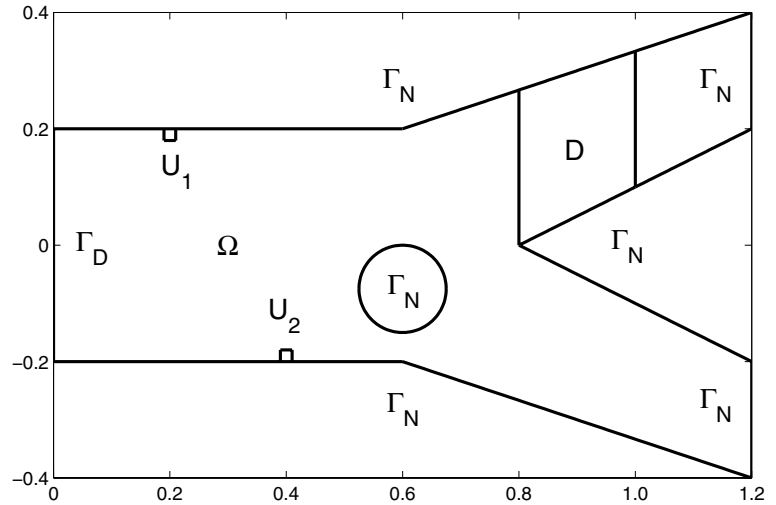

(a)

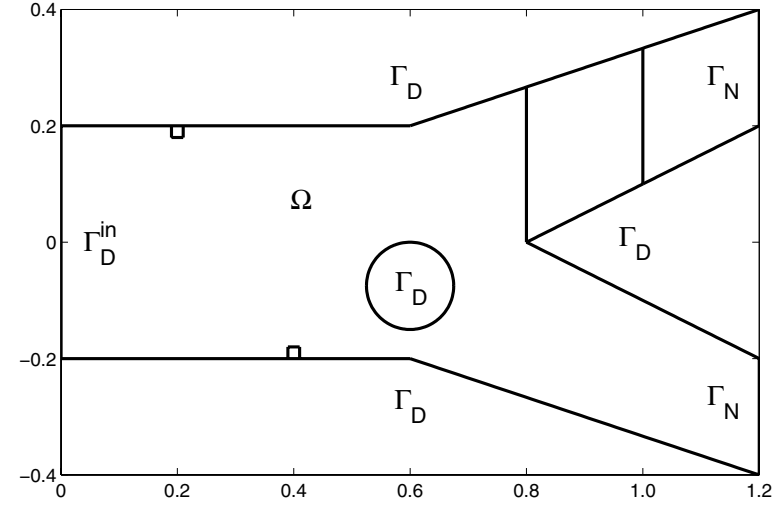

(b)

FiguRE 4. Test 1. Reference domain for the control problem. We report the boundary conditions for the advection-diffusion Equation (10) (a) and for the Stokes problem (64) (b).

where $\rho_{K}^{w}, \rho_{K}^{p}$ and $\rho_{K}^{u}$ are defined in Equations (55) and (61) (for the sake of simplicity, we have dropped the apex $(j)$ on the error indicators). Results are compared with those obtained on fine grids, that we consider an accurate guess of the exact solution.

\subsection{Test 1: water pollution}

Let us consider a first test case that is inspired to a problem of a water pollution. The optimal control problem consists in regulating the emission rates of pollutants (rising e.g. from refusals of industrial or agricultural plants) to keep the concentration of such substances below a desired threshold in a branch of a river.

We refer to the domain reported in Figure 4a, that could represent a river that bifurcates into two branches past a hole, which stands for, e.g., an island. Referring to Equation (10), we obtain the velocity field $\mathbf{V}$ as the solution of the following Stokes problem:

$$
\begin{cases}-\mu \Delta \mathbf{V}+\nabla p=\mathbf{0}, & \text { in } \Omega, \\ \mathbf{V}=\left(1-\left(\frac{y}{0.2}\right)^{2}, 0\right)^{T}, & \text { on } \Gamma_{D}^{\text {in }}, \\ \mathbf{V}=\mathbf{0}, & \text { on } \Gamma_{D}, \\ \mu \nabla \mathbf{V} \cdot \mathbf{n}-p \mathbf{n}=\mathbf{0}, & \text { on } \Gamma_{N},\end{cases}
$$

where $p$ stands for the pressure, while $\Gamma_{D}^{i n}, \Gamma_{D}$ and $\Gamma_{N}$ are indicated in Figure $4 \mathrm{~b}$. Adimensional quantities are used. Here the Stokes problem serves the only purpose to provide an appropriate velocity field for the advection-diffusion problem; since the latter governs our control problem, the analysis provided in Section 1 and Section 2 applies. Moreover, for the sake of simplicity, we adopt the method and the a posteriori error estimate (54) proposed in Section 3. In fact, this approach is not fully coherent, being the velocity field $\mathbf{V}$ computed numerically by means of the same grid adopted to solve the control problem, i.e. we consider $\mathbf{V}_{h}$ instead of $\mathbf{V}$.

For the Stokes problem we assume $\mu=0.1$, for which the Reynolds number reads $\mathbb{R} e \approx 10$; we solve the problem by means of linear finite elements with stabilization (see [16]), computed with respect to the same grid of the control problem. In Figure 5 we report the velocity field and its intensity as obtained by solving the Stokes problem.

For our control problem we assume $\nu=0.015, u=50$ in both the emission areas $U_{1}$ and $U_{2}$ and $z_{d}=0.1$ in the observation area $D$. The initial value of the control function, $u=50$, can be interpreted as the maximum rate of emission of pollutants (divided by the emission area), while the state variable $w$ stands for the pollutant 


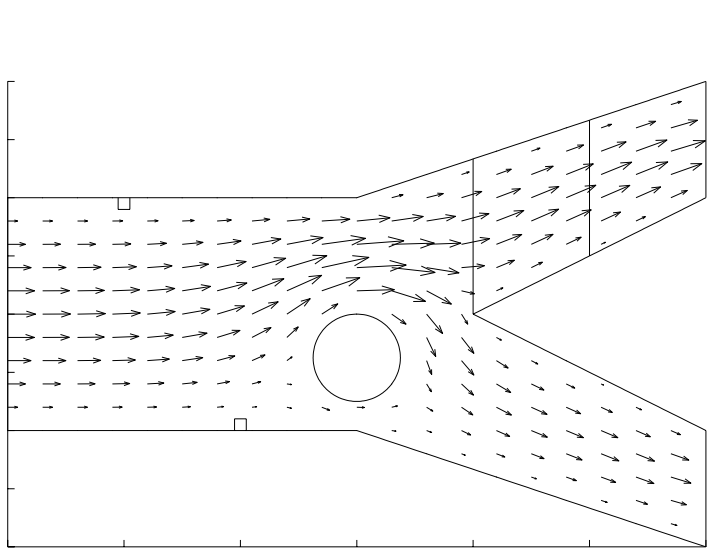

(a)

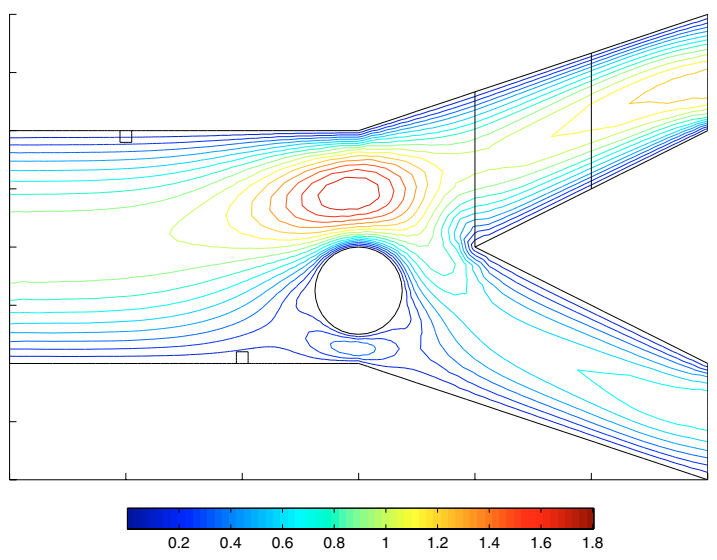

(b)

Figure 5. Test 1. Velocity field obtained by the Stokes problem: flow arrows (a) and velocity isolines (b).

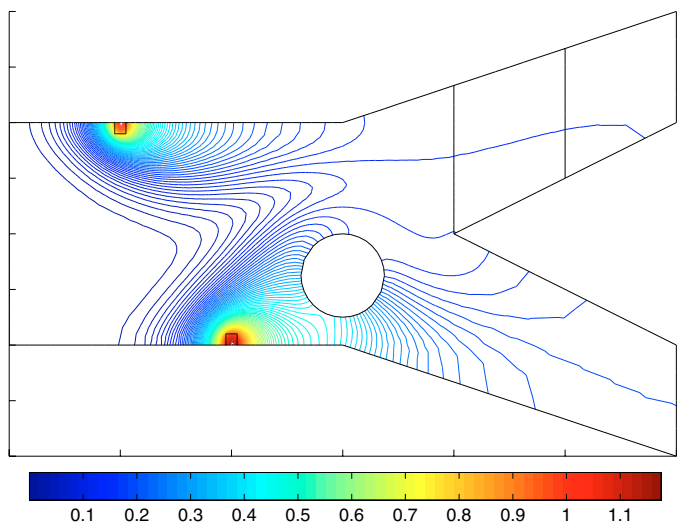

(a)

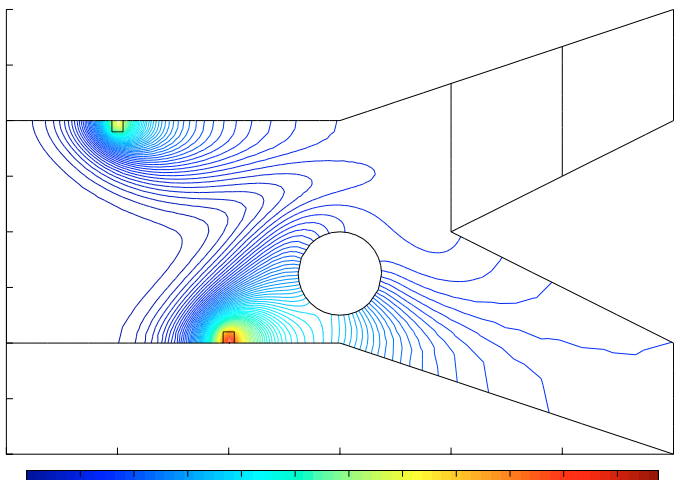

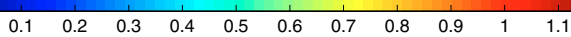

(b)

Figure 6. Test 1 . State solution at initial step (a) and at convergence $\left(\left\|J^{\prime}\right\| \leq 1.63 \times 10^{-7}\right)(\mathrm{b})$; at convergence it is $u_{1}=0.705 \times u_{\max }$, while $u_{2}=0.860 \times u_{\max }$.

concentration. We solve numerically the problem by means of the iterative method proposed in Section 1.1, with $\tau=500$ with respect to an initial grid of 212 triangular elements. Moreover, we perform grid adaption according to the a posteriori error estimate (54) and the method proposed in Section 3.

In Figure 6 we report the results of the optimal control problem: at the initial step, when the value of the state solution in the observation area is higher than the desired level $z_{d}$ (left), and at convergence $\left(\left\|J^{\prime}\right\| \leq 1.63 \times 10^{-7}\right)$ (right). The two emission sources $u_{1}$ and $u_{2}$ must be reduced with respect to the initial value $u_{\max }=50$. More precisely, we obtain that at convergence:

- the minimum value of the cost functional is $J=5.40 \times 10^{-9}$;

- the emission rates become $u_{1}=0.705 \times u_{\max }$ and $u_{2}=0.860 \times u_{\max }$.

In Figure 7 we show two grids adapted by means of the estimates on the error indicators $\eta_{D}$ and $\eta_{E}^{w p u}$; the grid that we would obtain by virtue of the error indicator $\eta_{E}^{w}$ results similar to that obtained by $\eta_{E}^{w p u}$. In Figure 8 we provide an error comparison for the cost functional and the value of $u_{2}$ on different grids and we prove 


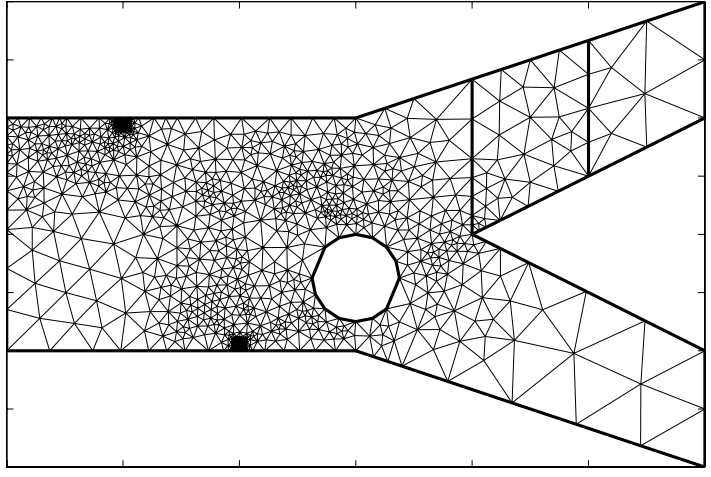

(a) $\eta_{D}$

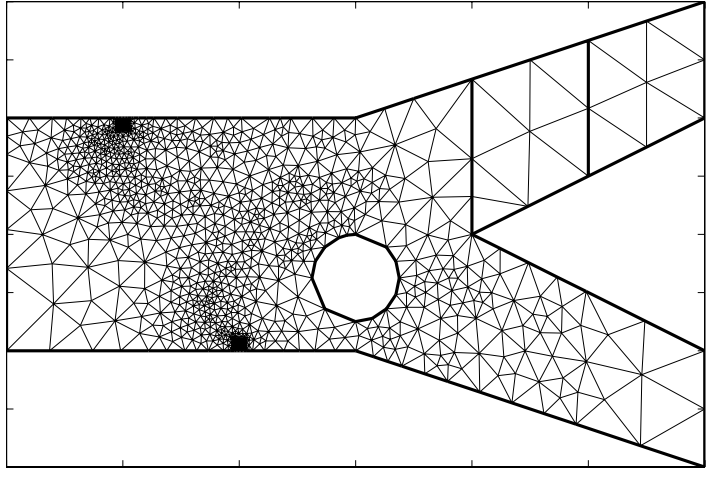

(b) $\eta_{E}^{w p u}$

Figure 7. Test 1. Adapted meshes (2700 elements) obtained using the error indicators $\eta_{D}$ (left) and $\eta_{E}^{w p u}$ (right); the error indicator $\eta_{E}^{w}$ produces an adapted grid very similar to that on the right.

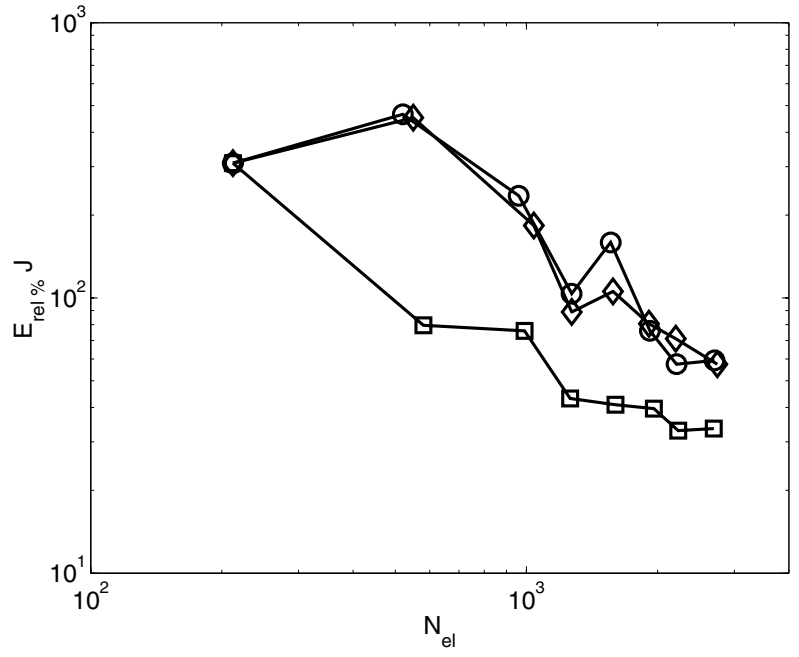

(a)

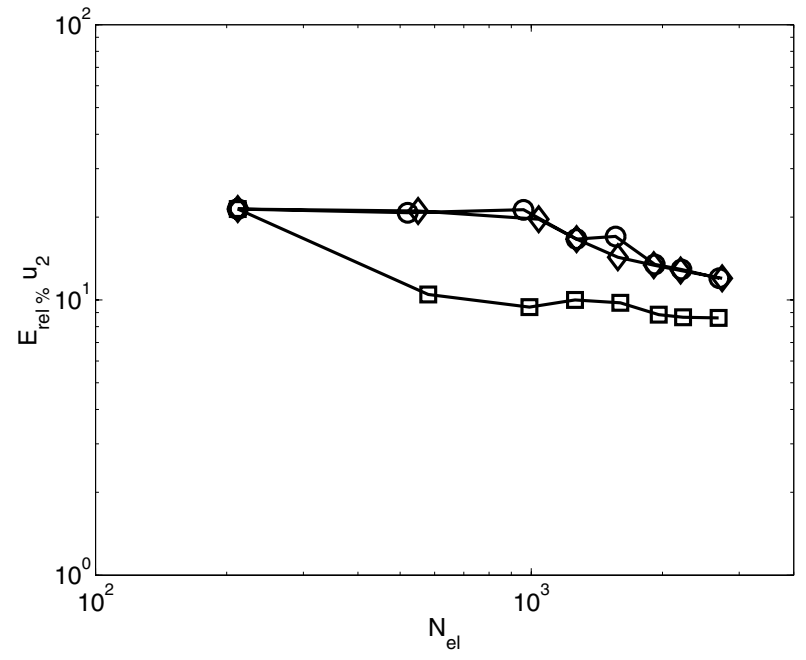

(b)

Figure 8. Test 1. Numerical results for adaptivity driven by the error indicators $\eta_{D}(\square)$, $\eta_{E}^{w}(\diamond)$ and $\eta_{E}^{w p u}(\mathrm{O})$ at convergence; we report relative errors (in percent) on cost functional $J$ (a) and on $u_{2}$ (b) with the number of elements $\left(N_{e l}\right)$ in $\log -\log$ scale; let us notice that analogous results hold also for the error on $u_{1}$.

the validity of the error indicator $\eta_{D}$ and the adaptive method. Similar results can be obtained for the error behavior of $u_{1}$. The adaptivity driven by the error indicator $\eta_{D}$ fits elements in those zones which are more relevant for the control problem (i.e. $\left\{U_{i}\right\}$ and $D$ ), while the error indicator $\eta_{E}^{w}$ governs the distribution of elements in those areas which are relevant for the state solution. Finally, the error indicator $\eta_{E}^{w p u}$ fits elements in those areas which are relevant for both the state and adjoint equations (like e.g. in areas where sources are located); however these contributions to error indicator are not balanced and, typically, the state error component dominates the other ones. This explain why $\eta_{E}^{w p u}$ and $\eta_{E}^{w}$ generate similar grids. Let us notice 


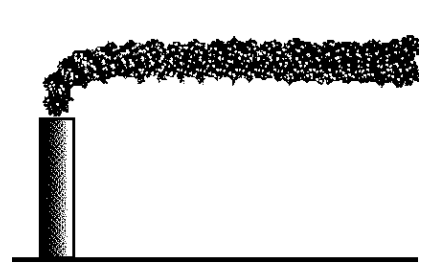

(a) stable

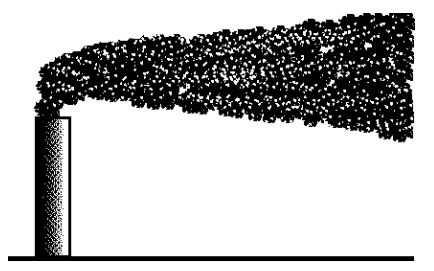

(b) neutral

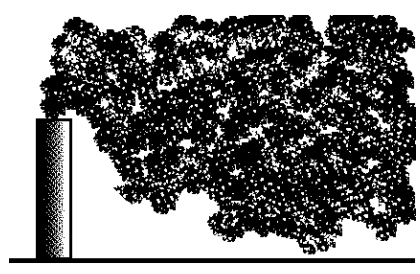

(c) instable

Figure 9. Test 2. Atmospheric stability conditions: emission chimney behavior in a wind field.

that in lieu of the exact solution, we use a numerical one, obtained with a grid with about 8000 elements, with elements properly distributed all over the domain.

\subsection{Test 2: air pollution}

The evolution of pollutant concentration in atmosphere is a complex phenomenon which depends on the pollutant type, the air composition (presence of other pollutants) and, of course, the specific meteorological situation (see [6]). If we consider particular pollutants, such as the sulfur oxides $\left(\mathrm{SO}_{2}\right)$, we can admit that in urban scales (see [6]) the concentration behavior can be modelled by advection-diffusion equations, neglecting, with acceptable accuracy, the reactive terms. Moreover we consider a stationarity frame, for which the advection-diffusion equation (10) can be assumed to model the pollutant behavior in air and the optimal control setting presented in Section 1 can be adopted. Let us notice that Equation (10) models the diffusion and transport of pollutant at the emission height, i.e. in a plane $x-y$ parallel to soil at height $H$. Modelling air motion is a very complex matter, especially in the planetary boundary layer (see [18]), depending on a broad range of factors, such as: soil orography, type of soil (urban or rural areas), pressure gradients, ground heating and Coriolis force. For this reason air motion is modelled by means of the medium wind field, plus terms taking into account the turbulent diffusion, which can be related to atmospheric stability (stability classes are typically subdivided as stable, neutral and instable; see Fig. 9 and [6]). In the contest of Equation (10), as reported in [6], the vector $\mathbf{V}$ takes into account the medium wind field, while the diffusivity term $\nu$ represents the turbulent diffusion. The molecular diffusivity is much smaller and can be neglected. As we have previously anticipated, Equation (10) describes the transport and diffusion of the pollutant in a plane, while a complete model should take into account 3D phenomena. We can adopt a quasi-3D model if we consider the pollutant concentration $w(x, y, z)$ depending on the concentration at the emission height (which is the solution of Eq. (10)) multiplied by a function $\pi(x, y, z)$ which takes into account the distribution of the concentration with the respect to the vertical coordinate $z$. The function $\pi$ can be generated by exploiting the analogy with a Gaussian model (see $[6]$ ). The latter, by assuming a constant wind field, provides the pollutant concentration in a parallelepipedal domain (3D) emitted by a unique source:

$$
\pi(x, y, z):=\mathrm{e}^{-\frac{1}{2}\left(\frac{z-H}{\sigma_{z}}\right)^{2}}+\mathrm{e}^{-\frac{1}{2}\left(\frac{z+H}{\sigma_{z}}\right)^{2}},
$$

where $\sigma_{x y}$ and $\sigma_{z}$ are the dispersion coefficients, which account for atmospherical stability class and soil orography (see [6]). For example, considering neutral atmospherical conditions and urban and rural soil, we have:

$$
\left\{\begin{array}{l}
\sigma_{x y}=0.120 r(1+0.00025 r)^{-\frac{1}{2}},[m] \\
\sigma_{z}=0.040 r(1+0.00020 r)^{-\frac{1}{2}},[m]
\end{array}\right.
$$

being $r$ the radial coordinate with origin in the emission source.

Our target consists in regulate the emission rates form $N$ chimneys $U_{i}$ to keep the pollutant concentration below a desired level in an observation area $D$, e.g. a town. More particularly, we refer to a domain such as 


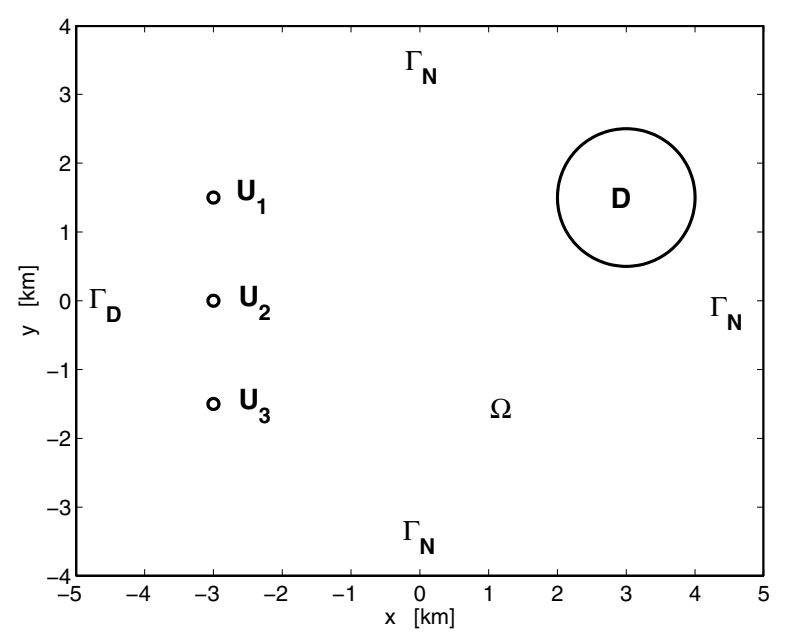

Figure 10. Test 2. Reference domain for emission control problem in air.

the one reported in Figure 10, for which we consider the optimal control setting presented in Section 1. To this aim we make the following assumptions:

- pollutant concentration distribution is governed by $2 D$ linear transport-diffusion equation (Eq. (10)) in non-conservation form, in the plane $x-y$, at effective chimney height; the concentration distribution with respect to $z$ is described by the Gauss model, according to the function $\pi$ in equation (65);

- turbulent diffusion coefficients depending on radial coordinate with origin at the emission zone $(\nu=\nu(r)$ );

- according to Figure $10, \mathbf{V}=V \hat{\mathbf{x}}$;

- emission areas $U_{i}$ are approximately at the same distance from the observation area $D$, in order to consider the same diffusion coefficient $\nu=\frac{\sigma_{x y}^{2}}{2 r} V$;

- all the emission plants stand at the same height $H$.

According to these assumptions we can adopt, for the optimal control problem, the same setting presented in Section 1.2, in which $w$ is the pollutant concentration at the emission height $H$ and $u=\sum_{i=1}^{N} u_{i} \chi_{U_{i}}$ is the emission rate (control function) (normalized with respect to an appropriate emission volume). We assume in Equation (11):

$$
g(x, y)=2 \mathrm{e}^{-\frac{1}{2}\left(\frac{H}{\sigma_{z}(x, y)}\right)^{2}},
$$

which is the projection term of pollutant concentration at soil, and $z_{d}=w_{d}^{0}$, which stands for the desired pollutant concentration at soil in the observation area $D$ (let us notice that for $\mathrm{SO}_{2}$ the attention level is $125 \mu \mathrm{g} / \mathrm{m}^{3}$, while the alarm level is $250 \mu \mathrm{g} / \mathrm{m}^{3}$; see [6]).

For our test case we refer to the domain reported in Figure 10 and we assume neutral atmospheric conditions and $\mathrm{SO}_{2}$ as pollutant, for which we take $100 \mu \mathrm{g} / \mathrm{m}^{3}$ as target concentration level $\left(w_{d}^{0}\right)$; this value is in general lower than both attention and alarm levels, however it may account for preexistent pollutant level in the domain. We assume that the maximum emission rate for chimneys is $u_{\max }=800 \mathrm{~g} / \mathrm{s}$ at effective height $H=100 \mathrm{~m}$ in a constant wind field $(V=2.5 \mathrm{~m} / \mathrm{s})$. The control function $u$ is the rate of emission normalized with respect to an appropriate emission volume. We solve numerically both the state and adjoint equations by the Galerkin-FE linear method on an initial grid of 808 elements. The steepest-descent iterative method is used to solve the control problem, for which we assume a relaxation parameter $\tau=800$. The grid adaptivity procedure is carried out using the estimate (54) and the method proposed in Section 3; we will compare the results provided by the error indicator $\eta_{D}$ (see Eq. (54)) with those obtained by virtue of the estimates (62) and (63).

We report pollutant concentration results in Figure 11: at the initial step (top), when the concentration in observation area is far superior than the admitted limit, and then at convergence of the optimization iterative 


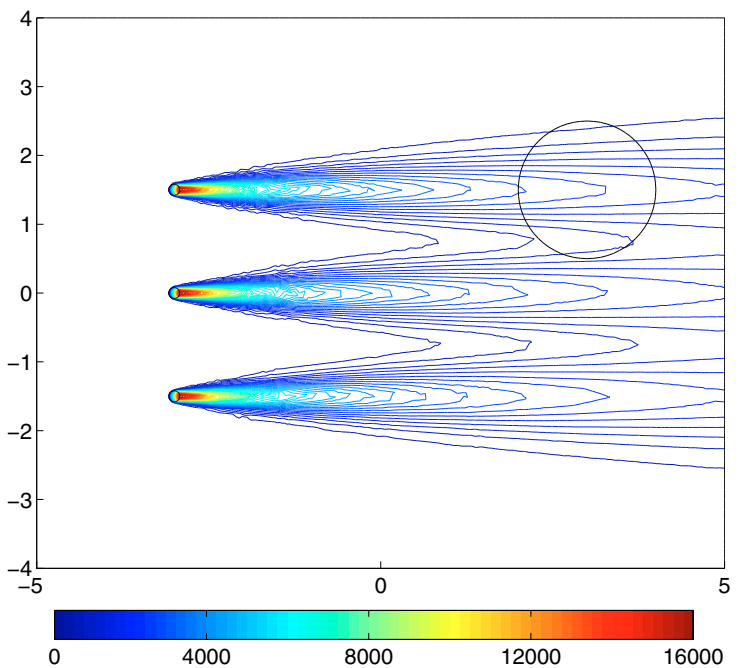

(a)

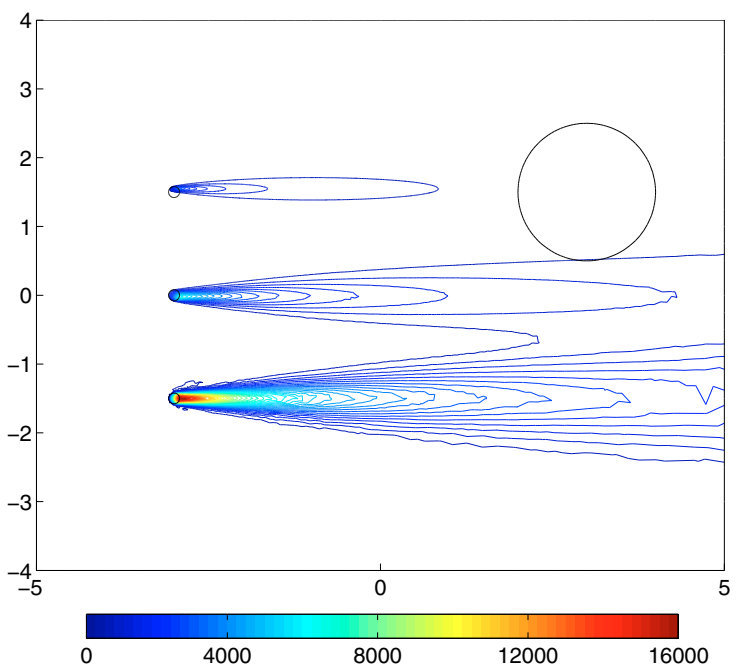

(c)

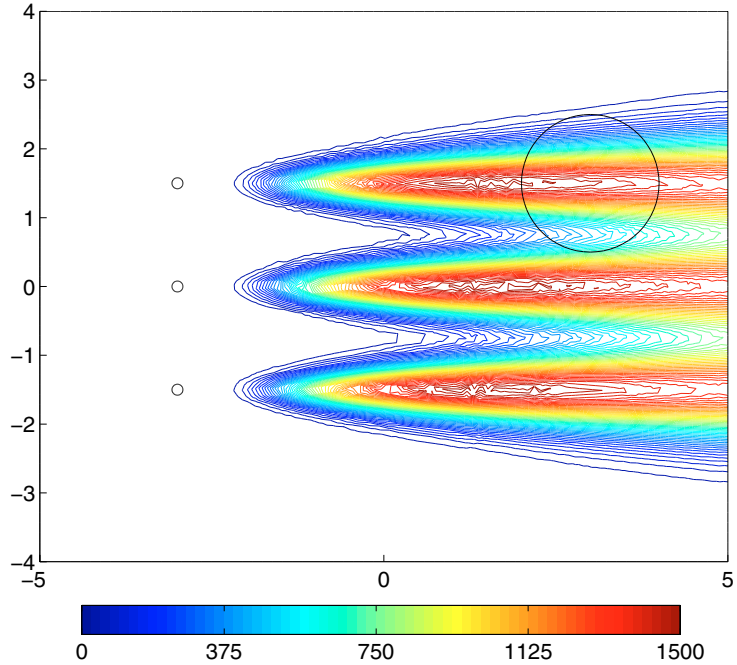

(b)

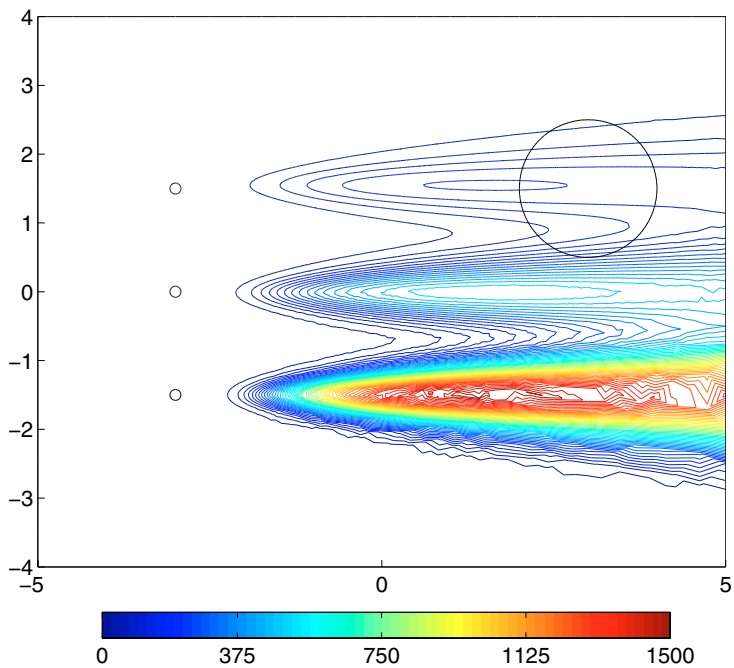

(d)

Figure 11. Test 2. State solutions at initial step and at convergence $\left(\left\|J^{\prime}\right\| \leq 1.8 \times 10^{-8}\right)$; pollutant initial concentrations $\left[\mu \mathrm{g} / \mathrm{m}^{3}\right]$ at effective height (a) and at soil (b) and, at convergence, in height (c) and at soil (d).

process (bottom). We observe that the emissions in the first and the second industrial complexes must be reduced, while the control problem does not involve appreciably the emissions of the third one. We report the results of the optimal control problem (at convergence when $\left\|J^{\prime}\left(p_{h}^{j}, u_{h}^{j}\right)\right\| \leq 1.8 \times 10^{-8}$, for the adimensional problem) for some interesting quantities:

- the minimum cost functional is $J=2.50 \times 10^{-9}$ (for the adimensional problem);

- the emission rates become $u_{1}=0.0858 \times u_{\max }, u_{2}=0.325 \times u_{\max }$ and $u_{3}=1.00 \times u_{\max }$;

- the maximum value of the concentration of $\mathrm{SO}_{2}$ in the observation area is $w_{\max }^{0}=187 \mu \mathrm{g} / \mathrm{m}^{3}$, which is lower than the alarm level. 


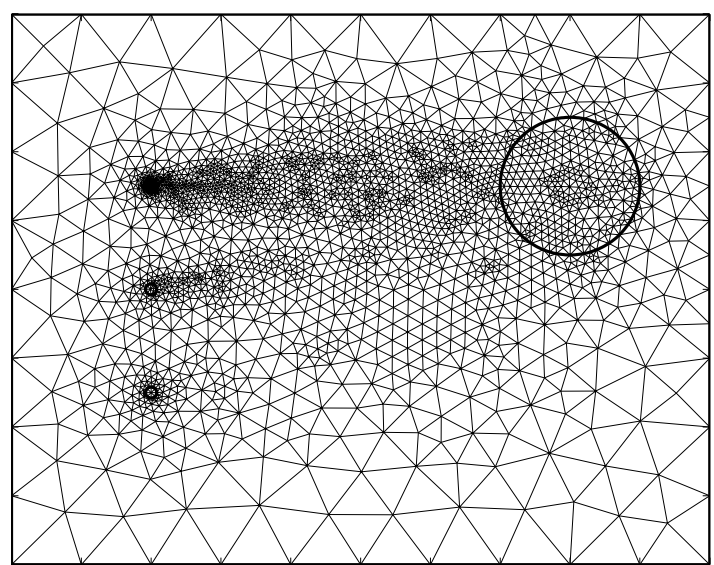

(a) $\eta_{D}$

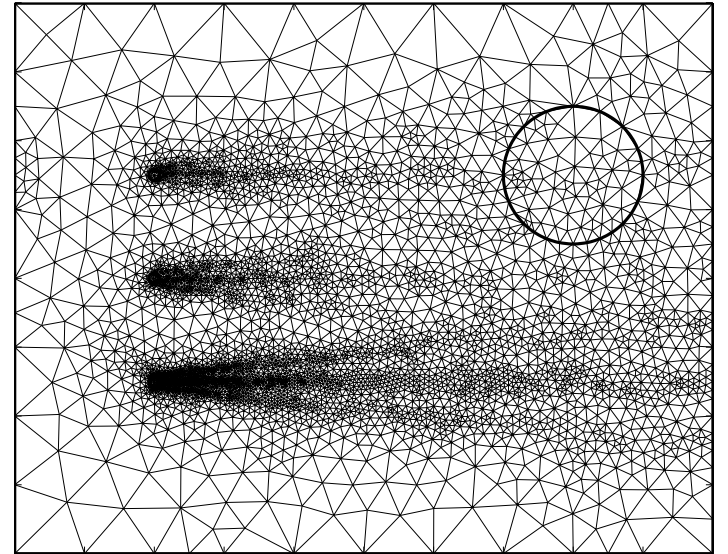

(b) $\eta_{E}^{w p u}$

Figure 12. Test 2. Adapted meshes (11000 elements) obtained using the error indicators $\eta_{D}$ (left) and $\eta_{E}^{w p u}$ (right); the error indicator $\eta_{E}^{w}$ produces an adapted grid very similar to that at the right.

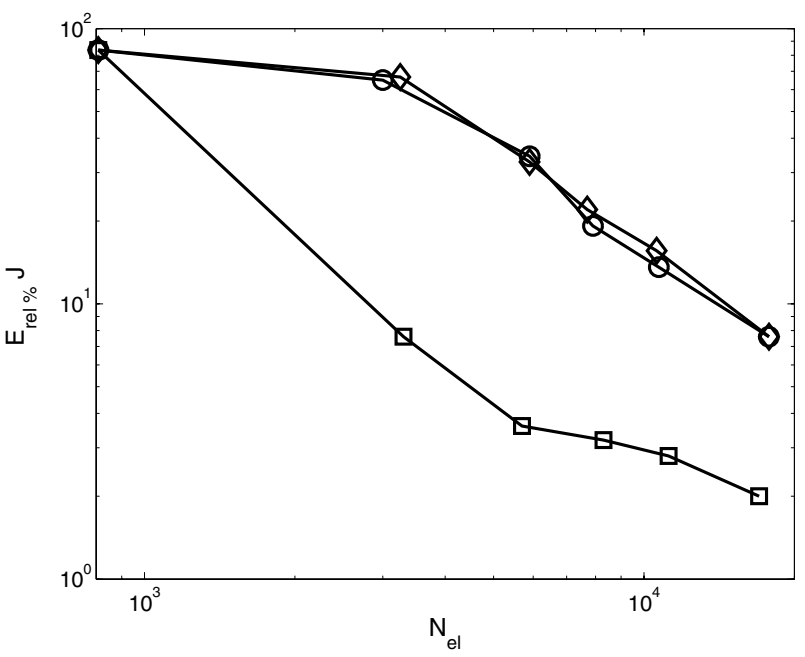

(a)

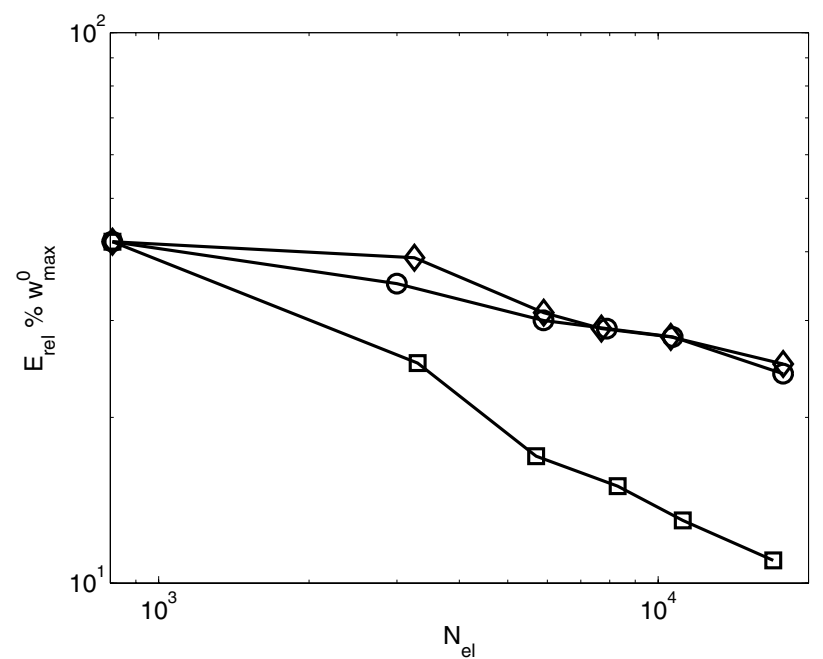

(b)

Figure 13. Test 2. Numerical results for adaptivity driven by the error indicators $\eta_{D}(\square)$, $\eta_{E}^{w}(\diamond)$ and $\eta_{E}^{w p u}(\mathrm{O})$ at convergence; we report relative errors (in percent) on cost functional $J$ (a) and on $w_{\max }^{0}(\mathrm{~b})$ with the number of elements $\left(N_{i t}\right)$ in $\log -\log$ scale.

In Figure 12 we show two grids adapted by virtue of the estimates on the error indicators $\eta_{D}$ and $\eta_{E}^{w p u}$; the grid that we would obtain by means of the error indicator $\eta_{E}^{w}$ does not differ significantly from that obtained using $\eta_{E}^{w p u}$. In Figure 13 we show an error comparison for the cost functional and the value of $w_{\max }^{0}$ on different grids, for which we prove the validity of the error indicator $\eta_{D}$ and the adaptive method. In particular, the adaptivity driven by the error indicator $\eta_{D}$ leads to a distribution of elements in those domain zones involved in the control problem (i.e. $\left\{U_{i}\right\}$ and $D$ ), whereas the error indicator $\eta_{E}^{w}$ governs the distribution of elements 


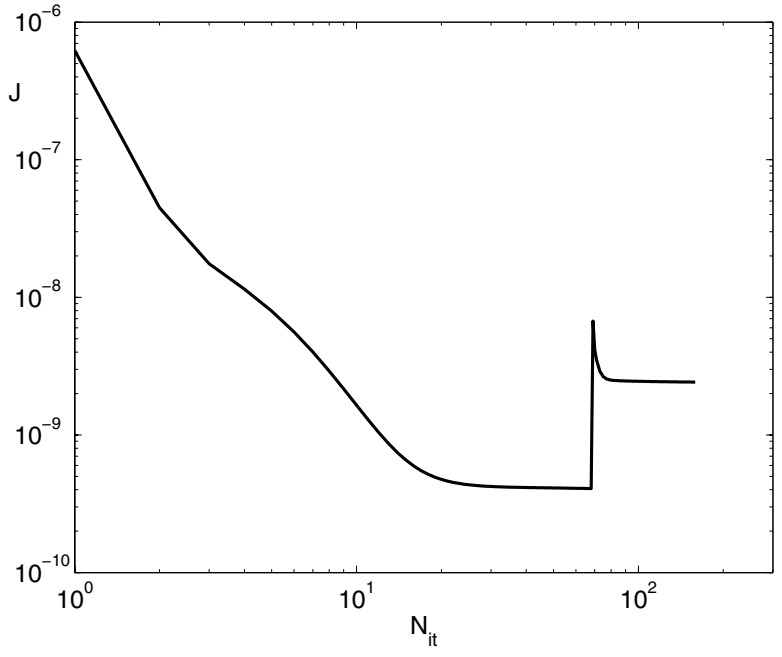

(a)

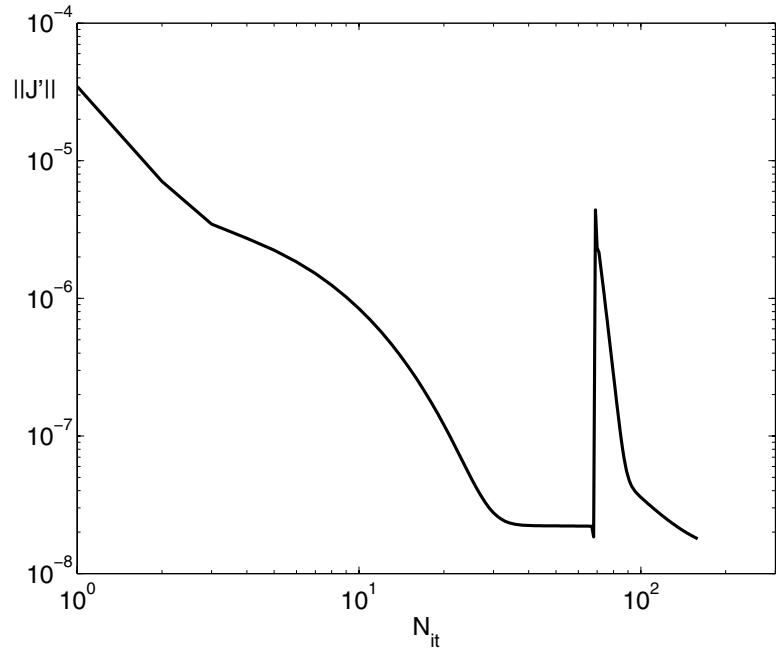

(b)

Figure 14. Test 2. Functional cost $J$ (a) and its derivative $\left\|J^{\prime}\right\|=\|\nabla \mathcal{L}\|$ (b) versus number of iterations $N_{i t}$ (in $\log -\log$ scale). Numerical adaptivity and grid refinement are based on the error indicator $\eta_{D}$; the final grid is composed of about 10500 elements.

in the most crucial areas for the state solution. Similarly, the error indicator $\eta_{E}^{w p u}$ fits elements in those areas which are relevant for the state and adjoint equations and where the derivative of the cost functional is defined, without balancing their contribution on the control problem. As already mentioned, $\eta_{E}^{w}$ generates a grid quite similar to that obtained by this last estimate. Let us notice that we consider as exact the solution that is actually computed using a grid with more than 80000 elements. In Figure 14 we report the behavior of the cost functional $J$ and the derivative of the cost functional (in the appropriate norm, $\left\|J^{\prime}\right\|$ ) versus the number of iterations. We recall that the latter is equivalent to the gradient of the Lagrangian functional. The jumps occur in correspondence with grid refinement.

\section{Conclusions}

In this work we have proposed a general approach to linear control problems governed by an advectiondiffusion state equation, dealing with both the theoretical aspects and the numerical ones. Particularly we have introduced the concept of the stabilized Lagrangian to avoid numerical instabilities, providing a valid tool to afford coherently control problems governed by advection-diffusion equations. On this basis we have proposed an error estimator dealing with the error on the cost functional, for which we have provided a separation of the discretization error from the iteration error. The estimator of the discretization error is used in the field of an adaptive method to refine the grid. The effectiveness of the proposed methods is proved on two numerical tests.

The methods proposed in this work can be easily extended to the case of control problems governed by advection-diffusion-reaction equations. Moreover the proposed approach can be conveniently applied to problems of real life interest, e.g. to pollution control problems in the field of environmental engineering. 
Acknowledgements. We are sincerely grateful to Prof. V.I. Agoshkov, Ing. G. Rozza, Ing. M. Restelli and Dr. L. Bonaventura for their advise and help. We acknowledge the Italian MIUR for the partial financing under the project COFIN03 "Numerical Modelling for Scientific Computing and Advanced Applications" and the École Polytechnique

Fédérale de Lausanne (EPFL).

\section{REFERENCES}

[1] V.I. Agoshkov, Optimal Control Methods and Adjoint Equations in Mathematical Physics Problems. Institute of Numerical Mathematics, Russian Academy of Science, Moscow (2003).

[2] A.K. Aziz, J.W. Wingate and M.J. Balas, Control Theory of Systems Governed by Partial Differential Equations. Academic Press, New York (1971).

[3] R. Becker and R. Rannacher, An optimal control approach to a posteriori error estimation in finite element methods. Acta Numer. 10 (2001) 1-102.

[4] R. Becker, H. Kapp and R. Rannacher, Adaptive finite element methods for optimal control of partial differential equations: basic concepts. SIAM J. Control Optim. 39 (2000) 113-132.

[5] M. Braack and A. Ern, A posteriori control of modelling errors and Discretization errors. SIAM Multiscale Model. Simul. 1 (2003) 221-238.

[6] G. Finzi, G. Pirovano and M. Volta, Gestione della Qualità dell'aria. Modelli di Simulazione e Previsione. Mc Graw-Hill, Milano (2001)

[7] L. Formaggia, S. Micheletti and S. Perotto, Anisotropic mesh adaptation in computational fluid dynamics: application to the advection-diffusion-reaction and the Stokes problems. Appl. Numer. Math. 51 (2004) 511-533.

[8] A.N. Kolmogorov and S.V. Fomin, Elements of Theory of Functions and Functional Analysis. V.M. Tikhomirov, Nauka, Moscow (1989).

[9] R. Li, W. Liu, H. Ma and T. Tang, Adaptive finite element approximation for distribuited elliptic optimal control problems. SIAM J. Control Optim. 41 (2001) 1321-1349.

[10] J.-L. Lions, Optimal Control of Systems Governed by Partial Differential Equations. Springer-Verlag, New York (1971).

[11] W. Liu and N. Yan, A posteriori error estimates for some model boundary control problems. J. Comput. Appl. Math. 120 (2000) 159-173.

[12] W. Liu and N. Yan, A Posteriori error estimates for distribuited convex optimal control problems. Adv. Comput. Math. 15 (2001) 285-309.

[13] B. Mohammadi and O. Pironneau, Applied Shape Optimization for Fluids. Clarendon Press, Oxford (2001).

[14] M. Picasso, Anisotropic a posteriori error estimates for an optimal control problem governed by the heat equation. Int. J. Numer. Method PDE (2004), submitted.

[15] O. Pironneau and E. Polak, Consistent approximation and approximate functions and gradients in optimal control. SIAM J. Control Optim. 41 (2002) 487-510.

[16] A. Quarteroni and A. Valli, Numerical Approximation of Partial Differential Equations. Springer-Verlag, Berlin and Heidelberg (1994).

[17] J. Sokolowski and J.P. Zolesio, Introduction to Shape Optimization (Shape Sensitivity Analysis). Springer-Verlag, New York (1991).

[18] R.B. Stull, An Introduction to Boundary Layer Meteorology. Kluver Academic Publishers, Dordrecht (1988).

[19] F.P. Vasiliev, Methods for Solving the Extremum Problems. Nauka, Moscow (1981).

[20] D.A. Venditti and D.L. Darmofal, Grid adaption for functional outputs: application to two-dimensional inviscid flows. J. Comput. Phys. 176 (2002) 40-69.

[21] R. Verfürth, A Review of A Posteriori Error Estimation and Adaptive Mesh-Refinement Techniques. Wiley, Teubner (1996). 